\title{
True and false recognition memories of odors induce distinct neural signatures
}

\section{Jean-Pierre Royet ${ }^{1,2}$, Léri Morin-Audebrand ${ }^{3,4}$, Barbara Cerf-Ducastel ${ }^{5}$, Lori Haase ${ }^{5}$, Sylvie Issanchou ${ }^{3}$, Claire Murphy ${ }^{5}$, Pierre Fonlupt ${ }^{6}$, Claire Sulmont-Rossé ${ }^{3}$ and Jane Plailly ${ }^{1}$}

\author{
INSERM, U1028, UMR5292 CNRS, Lyon Neuroscience Research Center, Université Lyon, Lyon, France \\ 2 CERMEP, Neurological Hospital, Lyon, France \\ ${ }^{3}$ Centre des Sciences du Goût et de I'Alimentation, UMR6265 CNRS, UMR1324 INRA, Université de Bourgogne, Dijon, France \\ ${ }^{4}$ Institute of Life Technologies, University of Applied Sciences Valais, Sion, Switzerland \\ ${ }^{5}$ Lifespan Human Senses Laboratory, Department of Psychology, San Diego State University, San Diego, CA, USA \\ ${ }^{6}$ Dynamique Cérébrale et Cognition, INSERM, U280, University Lyon1, Lyon, France
}

\section{Edited by:}

Hans-Jochen Heinze, University of

Magdeburg, Germany

\section{Reviewed by:}

Leslie J. Carver, University of California, USA

Mercedes Atienza, University Pablo de Olavide, Spain

\section{${ }^{*}$ Correspondence:}

Jean-Pierre Royet, INSERM, U1028, UMR5292 CNRS, Lyon Neuroscience Research Center, University Lyon1, Olfaction: From Coding to Memory, 50 Cedex 07, France.

e-mail: royet@olfac.univ-lyon1.fr Avenue Tony Garnier, 69366 Lyon

Neural bases of human olfactory memory are poorly understood. Very few studies have examined neural substrates associated with correct odor recognition, and none has tackled neural networks associated with incorrect odor recognition. We investigated the neural basis of task performance during a yes-no odor recognition memory paradigm in young and elderly subjects using eventrelated functional magnetic resonance imaging. We explored four response categories: correct (Hit) and incorrect false alarm (FA) recognition, as well as correct (CR) and incorrect (Miss) rejection, and we characterized corresponding brain responses using multivariate analysis and linear regression analysis. We hypothesized that areas of the medial temporal lobe were differentially involved depending on the accuracy of odor recognition. In young adults, we found that significant activity in the hippocampus and the parahippocampal gyrus was associated with correct (true) recognition of odors, whereas the perirhinal cortex was associated with FAs and Misses. These findings are consistent with literature regarding hypothetical functional organization for memory processing. We also found that for correct recognition and rejection responses, the involvement of the hippocampus decreased when memory performances improved. In contrast to young individuals, elderly subjects were more prone to false memories and exhibited less specific activation patterns for the four response categories. Activation in the hippocampus and the parahippocampal gyrus was positively correlated with response bias scores for true and false recognition, demonstrating that conservative subjects produced an additional search effort leading to more activation of these two medial temporal lobe regions. These findings demonstrate that correct and incorrect recognition and rejection induce distinct neural signatures.

Keywords: odor recognition memory, true memory, false memory, memory score, bias score, age effect, fMRI, multivariate analysis

\section{INTRODUCTION}

Recognition memory, a type of long-term memory, has classically been subdivided into two components: recollection and familiarity (Jacoby, 1991). Recollection refers to memory retrieval accompanied by the recovery of specific contextual details, whereas familiarity refers to the feeling that an item has been previously encountered in the absence of confirmatory contextual information (Daselaar et al., 2006). These two processes have been conceptualized from threshold- and signal-detection-based models that have widely dominated recognition memory theory since the 1950s (Lockhart and Murdock, 1970; Snodgrass and Corwin, 1988). This dual-process theory assumes that recollection is a threshold process and that familiarity is a signal-detection process (Yonelinas, 1994).

A classic method used to investigate recognition memory is a yes-no-recognition task, in which a first set of stimuli is presented (encoding), followed by a second set of stimuli comprised of the initial stimuli with novel stimuli interleaved (retrieval; Roediger and McDermott, 1995). Subjects must indicate whether stimuli in the second set have been previously presented (old) or not (new).
From these two experimental conditions, four response categories can be defined: true memory (correct recognition, Hit) and Miss when the old items are respectively accurately recognized and incorrectly rejected, and correct rejection (CR) and false recognition (false alarm, FA) when the new items are respectively correctly rejected and incorrectly recognized. In the framework of the signaldetection theory, a memory score reflects the subject's ability to discriminate between old and new items, and a response bias score reflects the decision rule adopted when responding (Lockhart and Murdock, 1970; Snodgrass and Corwin, 1988). One can further hypothesize that Hit responses rely more on a recollection process, while FAs rely more on a familiarity process (Yonelinas et al., 1996; Wixted and Stretch, 2004).

Since the 1990s, neuroimaging has greatly contributed to the understanding of recognition memory processes. Most researchers have focused on recognition accuracy by comparing only Hit and FA responses (Cabeza et al., 2001; Slotnick and Schacter, 2004; GaroffEaton et al., 2006). Differences in brain activation have primarily been reported in the fronto-parietal and medial temporal regions 
of the brain (Daselaar et al., 2001; Leube et al., 2003; Heun et al., 2004; Garoff-Eaton et al., 2006). For example, Cabeza et al. (2001) found a dissociation between two distinct medial temporal regions with word stimuli: the hippocampus contributed to the generation of both Hit and FA responses, while the parahippocampal gyrus displayed greater activation for Hits than FAs. However, little is known about the neural networks of rejection accuracy (CR and Miss).

To study the neural networks associated with different response categories, the use of classic methods for analyzing cerebral images lead to perform several contrasts. Thus, the majority of functional imaging studies have implemented univariate analysis that can provide for statistical inferences about brain regions implicated in specific tasks. However, multivariate analysis can characterize brain responses in terms of functionally connected and distributed systems, suggesting that an entire network is engaged by a task. Therefore, univariate and multivariate analyses must be regarded as complementary approaches. Another aspect never investigated as far as we know, but that can guided us in the understanding of recognition memory mechanisms, is examination of the neural networks differentially activated as a function of subjects' intrinsic characteristics and their responses.

In olfaction, no neuroimaging study has attempted to dissociate the processes related to true and false memory and correct and incorrect rejection (Savic et al., 2000; Royet et al., 2001; Gottfried et al., 2004; Savic and Berglund, 2004; Cerf-Ducastel and Murphy, 2006, 2009; Plailly et al., 2007). The present study aimed to shed light on the neural underpinnings of odor recognition memory and its misdeeds by exploring the processes associated with the four response categories. We extracted regions in which activation significantly differed as a function of different response categories, and performed region-of-interest analyses for areas known to be involved in olfactory and memory processes, such as the piriform cortex and the hippocampus. We used multivariate analyses of covariance (MANCOVA) and canonical variate analysis (CVA) to highlight the neural networks associated with the different response categories. We then characterized these networks as a function of memory and bias scores that defined the intrinsic characteristics of subjects. In order to achieve a wide response range, we investigated an elderly population that had previously demonstrated proneness for false memories, particularly for odors (Murphy et al., 1997; Cabeza et al., 2004; Dennis et al., 2008; Cerf-Ducastel and Murphy, 2009), additionally to a young cohort.

\section{MATERIALS AND METHODS PARTICIPANTS}

Sixteen young [ 7 men; age: $27.14 \pm 5.27$ years (mean \pm SD); range: $21.90-37.30$ ] and 22 elderly ( 11 men; age $68.64 \pm 3.29$ years; range: 65.00-74.76) right-handed participants without known olfactory impairments, rhinal disorders (colds, active allergies, a history of nasal-sinus surgery, or asthma), pregnancy, neurological disease, ferrous implants (e.g., pacemakers and cochlear implants), or claustrophobia participated in the study. Subjects were selected by olfactory ability with a forced-choice suprathreshold detection test (96.8\% correct in young subjects and $94.9 \%$ in elderly subjects) and mean duration of their breathing cycle [young: $3.75 \pm 0.56$, ranging from 3 to 5 s/cycle; elderly: $4.67 \pm 1.41$, ranging from 2.86 to 8.57 s/cycle; $\left.F_{(1,14)}=10.811, p=0.0054\right]$. This study was conducted in accordance with the Declaration of Helsinki. All subjects provided written informed consent as required by the local Institutional Review Board according to French regulations on biomedical experiments with healthy volunteers [Ethical Committee of CPP-Sud Est II (n CPP A 06-024), DGS2006-0226, May 11, 2006].

\section{OLFACTORY STIMULI}

One hundred and 30 odorants were used: 30 odorants for training purposes and 100 for the functional magnetic resonance imaging (fMRI) scanning session. The high number of stimuli ensured a sufficient number of Hit, Miss, CR, and FA responses.

For fMRI, odorants were distributed into 50 target (old) and 50 distractor (new) odorants (Table 1). Stimuli were counterbalanced by quality (e.g., floral, spicy, and fruity), and mean scores of intensity, hedonicity, and familiarity obtained from previous data (Royet et al., 2001). For old and new odorants, scores rated on a 0-10 scale were similar for intensity [old, $5.62 \pm 0.96$; new, $5.62 \pm 0.63$, $\left.F_{(1,41)}=0.426\right]$, hedonic valence $[4.23 \pm 1.59$ and $4.78 \pm 1.44$, respectively; $\left.F_{(1,41)}=1.621\right]$, and familiarity $[4.89 \pm 1.14$ and $5.34 \pm 1.04$, respectively; $\left.F_{(1,41)}=3.192\right]$.

The odorants were diluted in mineral oil (Sigma Aldrich, SaintQuentin-Fallavier, France) to a concentration of $10 \%$ in volume. For stimuli presentation, $5 \mathrm{ml}$ of this solution was absorbed into compressed polypropylene filaments inside a $100-\mathrm{ml}$ white polyethylene squeeze-bottle equipped with a dropper (Fisher Scientific, Illkirch, France).

\section{STIMULATING AND RECORDING MATERIALS}

The odorants were presented to the subjects using an airflow olfactometer, which allows the stimuli to be synchronized with breathing (Vigouroux et al., 2005). The stimulation equipment consisted of two modules: a non-ferrous (Duralumin ${ }^{\circledR}$ ) air-dilution injection head (placed in the magnet room) and the electronic component of the olfactometer (positioned outside the magnet room). Compressed air (10 l/min) was pumped into the olfactometer and delivered continuously through a standard oxygen mask positioned on the subject's face. At the beginning of an inspiration phase, an odorant was injected into the olfactometer by rapidly squeezing the odor bottle into the injection head, thereby transmitting the odorant to the mask. Information regarding the onset of stimulation was transmitted by optical fibers to analog-to-digital converters located outside the magnetically shielded room and powered by nickelcadmium batteries. Presentation timing was monitored using commercially available Presentation software (Neurobehavioral Systems, Inc., Albany, CA, USA) and was synchronized with the scanner.

Participant responses were acquired with a two key-press button box which provided logic signals. Breathing was recorded using polyvinyl-chloride foot bellows (Herga Electric Limited, Suffolk, UK) secured to the subject's abdomen with a cotton belt. Subject responses, breathing data, stimulation onset, and trigger signals from the MRI scanner were recorded online ( $100 \mathrm{~Hz}$ sampling rate) on a laptop equipped with a digital acquisition board I/O card (PCI-6527; National Instruments ${ }^{\circledR}$, Austin, TX, USA) using the LabVIEW software package (National Instruments ${ }^{\circledast}$ ). Data were further analyzed using custom routines created with Matlab (The Mathworks, Natick, MA, USA). 
Table 1 | List of target and distractor odorants.

\begin{tabular}{|c|c|c|}
\hline & Target & Distractor \\
\hline 1 & Acetophenone & Anchovy \\
\hline 2 & Apricot & Anise \\
\hline 3 & Bergamot & Apple \\
\hline 4 & Biscuit & Banana \\
\hline 5 & Blue cheese & Basil \\
\hline 6 & Bornyl acetate & Bay-tree \\
\hline 7 & 2-Bromophenol & Bitter almond \\
\hline 8 & Butan-1-ol & Bread \\
\hline 9 & Camphor & Buchu \\
\hline 10 & Caramel & Chocolate \\
\hline 11 & Carrot & Cinnamon \\
\hline 12 & Cauliflower & Citronella \\
\hline 13 & Celery & Clove \\
\hline 14 & Cherry & Coconut \\
\hline 15 & Coffee & Cypress \\
\hline 16 & Diacetyl & Ethyl acetate \\
\hline 17 & 1,4-Dichlorobutane & Eucalyptus \\
\hline 18 & Diethyl ether & Garlic \\
\hline 19 & Ethyl benzoylacetate & Geranium \\
\hline 20 & Ethyl nitrite & Grapefruit \\
\hline 21 & Ethyl propionate & (E)-Hex-2-enal \\
\hline 22 & Gardenia & (Z)-Hex-3-en-1-ol \\
\hline 23 & Guaiacol & Honey \\
\hline 24 & Hazelnut & Honeysuckle \\
\hline 25 & Hyacinth & Incense \\
\hline 26 & Jonquil & Isopropylacetate \\
\hline 27 & Melon & Jasmine \\
\hline 28 & Methyl acetate & Lavender \\
\hline 29 & Octan-2-ol & Lemon \\
\hline 30 & Onion & Licorice \\
\hline 31 & Parsley & Lily \\
\hline 32 & Peach & Lily of the valley \\
\hline 33 & Pepper & Mint \\
\hline 34 & Pin & Musk \\
\hline 35 & Plum & Nutmeg \\
\hline 36 & Potato & Octanal \\
\hline 37 & Sage & Oct-1-en-3-ol \\
\hline 38 & Scallop & Orange \\
\hline 39 & Smoked salmon & Passion fruit \\
\hline 40 & Tar & Patchouli \\
\hline 41 & Tarragon & Pear \\
\hline 42 & Tetradecanal & 2-Phenylpropianaldehyde DMA \\
\hline 43 & Tetrahydrothiophene & Prawn \\
\hline 44 & Lime tea & Raspberry \\
\hline 45 & Tobacco & Red currant \\
\hline 46 & Tomato & Rose \\
\hline 47 & Turpentine & Thyme \\
\hline 48 & Vine & Vanilla \\
\hline 49 & Wild strawberry & Vinegar \\
\hline 50 & Yeast & Violet \\
\hline
\end{tabular}

$D M A$, dimethyl acetal.
The amplitudes of the inspiratory and expiratory waveforms were estimated by integrating the curves respectively located on both sides of the baseline. The inspiratory volumes were computed for the first inspiratory cycle following each odor stimulation and these data were entered into a two-way (Group $\times$ Response category) analysis of variance (ANOVA) with repeated response category measurements using Statistica (StatSoft ${ }^{\natural}$, Tulsa, OK, USA).

\section{EXPERIMENTAL PROCEDURE}

Two functional runs were performed corresponding to encoding and retrieval sessions (Figure 2) separated by the structural image acquisition sequence $(10 \mathrm{~min})$. The 50 target odorants were presented in the encoding session and then interleaved with 50 distractor odorants in the retrieval session. Odorants were delivered according to an eventrelated fMRI design with a jittered interstimulus interval of $\sim 15 \mathrm{~s}$, depending on the participant's respiration. The order of presentation of odorants was randomized between participants for both sessions.

During the encoding session, participants indicated when they detected an odorant by pressing one button with their right hand. Since no landmark was provided to participants when stimulation was delivered, no-response was asked when they did not detect an odorant. For half of the subjects, the "detection" response was obtained with the index finger. For the other half of the subjects, it was obtained with the middle finger. They were not instructed about the objective of the next session. During the retrieval session, participants indicated whether or not they had already smelled the odorant in the first session. "Yes" and "no" responses were obtained with the index and the middle fingers, respectively, for $50 \%$ of participants and inversed for the other $50 \%$. General instructions were provided outside the scanner. The day before fMRI, subjects were trained outside the MR facility to breathe naturally and regularly without sniffing or holding their breath, to detect odors during inspiration while avoiding sniffing, and to rapidly provide responses (odor vs. no odor) using the key-press button box. Participants wore earplugs to protect them from scanner noise and kept their eyes closed during scanning.

\section{BEHAVIORAL DATA ANALYSIS}

Recognition memory performance was assessed using parameters issued from the signal-detection theory (Lockhart and Murdock, 1970). As a function of the experimental conditions (old or new

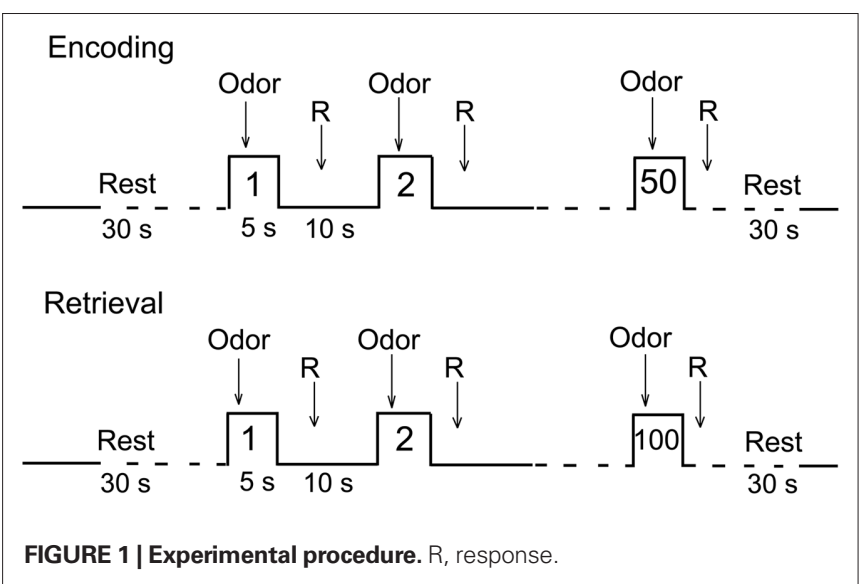



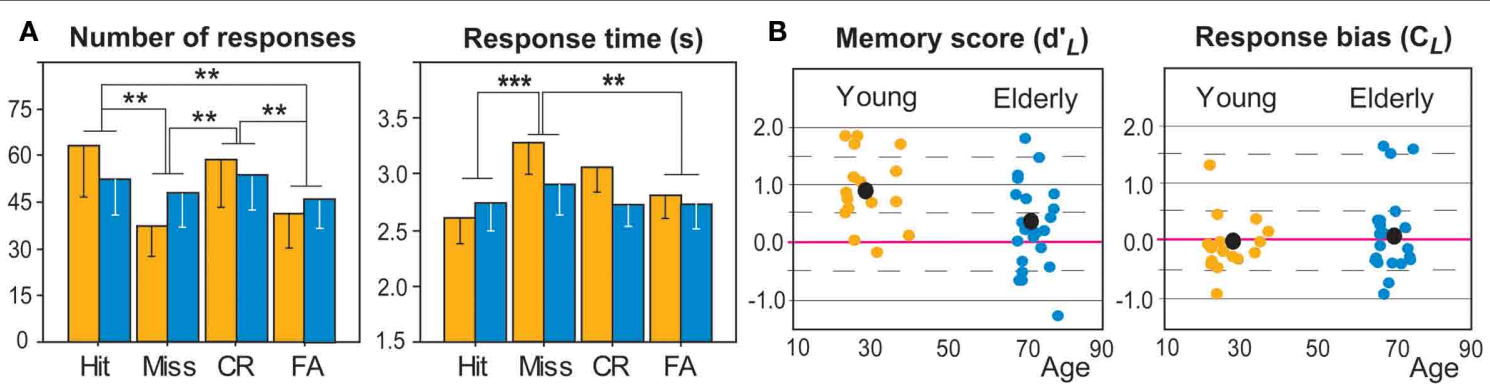

FIGURE 2 | Behavioral data in young (orange) and elderly (dark blue) subjects. (A) Number of responses (in percent of Hit and Miss relative to the number of old items and percent of FA and CR relative to the number of new items), and Responses times, as a function of four response categories. (B) Memory scores ( $\left.d^{\prime}{ }_{L}\right)$ and response bias scores $\left(C_{L}\right)$. Black dot, mean value; red line, zero value; ${ }^{*} p<0.05 ;{ }^{* *} p<0.01 ;{ }^{* *} p<0.001$.

odorants) and the subjects' behavioral answers (yes or no), the Hit, Miss, CR, and FA response categories were considered. Two parameters were calculated from the Hit and FA scores: memory score $\left(d_{L}^{\prime}\right)$ and response bias score $\left(C_{L}\right)$. Corwin (1989) previously described these calculations as follows:

$d_{L}^{\prime}=\ln \frac{H R(1-F R)}{F R(1-H R)}$

$C_{L}=0.5 \times \ln \frac{(1-F R)(1-H R)}{(H R \times F R)}$

where $H R$ represents the Hit rate $\left[(\mathrm{Hit}+0.5) /\left(N_{1}+1\right)\right]$, FR represents the false alarm rate $\left[(F A+0.5) /\left(N_{2}+1\right)\right]$, and $N_{1}$ and $N_{2}$ represent the number of old and new odorants, respectively, for which the subjects have provided an answer. Memory scores may be good or poor (positive and negative values, respectively). Response bias scores establish three individual attitudes. Subjects may be conservative (tending to respond "no" to an odor), neutral (responding "yes" or "no" with equal probability) or liberal (tending to respond "yes"; Snodgrass and Corwin, 1988) with positive, neutral or negative values, respectively.

\section{IMAGING PARAMETERS AND STATISTICAL ANALYSES Imaging parameters}

Images were acquired using a 1.5-Tesla MAGNETOM Sonata wholebody imager (Siemens medical ${ }^{\circledR}$, Erlangen, Germany) equipped with a four-channel circularly polarized head coil. For functional imaging, we obtained 26 interleaved, 4 -mm-thick axial slices using a T2* ${ }^{\star}$-weighted echo-planar sequence with the following parameters: repetition time $(\mathrm{TR})=2500 \mathrm{~ms}$, echo time $(\mathrm{TE})=50 \mathrm{~ms}$, flip angle $=80^{\circ}$, file-ofview $(\mathrm{FOV})=240 \mathrm{~mm} \times 240 \mathrm{~mm}$, and imaging matrix $=64 \times 64$ (voxel size: $3.8-\mathrm{mm} \times 3.8-\mathrm{mm} \times 4-\mathrm{mm}$ ). We collected 324 scans for the encoding session and 624 scans for the retrieval session. Between functional sessions, a high-resolution structural T1-weighted anatomical image (inversion-recovery 3D Gradient-Echo sequence, $1-\mathrm{mm} \times 1-\mathrm{mm} \times 1-\mathrm{mm})$ parallel to the bicommissural plane and covering the entire brain was acquired over $\sim 10 \mathrm{~min}$.

\section{fMRI data pre-processing and statistical analyses}

We processed all functional images using Statistical Parametric Mapping software (SPM2, Wellcome Department of Cognitive Neurology, London, UK; Friston et al., 1995a). For each subject, the first four volumes of each functional run were discarded to allow for T2* equilibration effects. Slices of each remaining volume were slice timing-corrected. All functional volumes were realigned to the median volume, co-registered to the anatomical image, spatially normalized to the Montreal Neurological Institute (MNI) standard brain (Friston et al., 1995b), and smoothed with an 8 - $\mathrm{mm} \times 8-\mathrm{mm} \times 10-\mathrm{mm}$ full-width half-maximum Gaussian kernel. Pre-processed data were statistically analyzed on a subjectby-subject basis using the general linear model. The regressors were modeled by convolving a neural model derived from the stimuli onsets with a hemodynamic response function (hrf).

For each subject, activity associated with five experimental conditions of interest (Hit, FA, CR, Miss, and non-answer) was modeled using boxcar predictors convolved with both the canonical hrf and its time-derivative (Friston et al., 1998; Hopfinger et al., 2000). A high-pass filter (with a cut-off frequency of $1 / 120 \mathrm{~Hz}$ ) was used to eliminate instrumental and physiological signal fluctuations at very low frequencies. Each trial was categorized on the basis of participants' answers (or absence of an answer) during the scanning session. Stimulus onset asynchronies were fixed at the time of odor delivery. Confounding factors (e.g., head motion) were included in the model. No participant moved more than $3 \mathrm{~mm}$ in any direction within or across runs. Thus, no data were eliminated in either age group due to motion artifacts. Random-effects analyses were performed to extrapolate statistical inferences at the population level, as described in the SPM2 software. Voxel-by-voxel single sample $t$-tests were performed for the response categories [Hit], [FA], [CR], and [Miss] to highlight activation that differed significantly from zero. Activations common to the four response categories were determined by a conjunction (intersection) of the four simple contrasts for both groups.

Next, to distinguish areas preferentially activated as a function of the four response categories and both groups, $12 t$-contrast maps were calculated by comparing Hit, FA, CR, and Miss items to each other, i.e., [Hit vs. FA], [Hit vs. CR], [Hit vs. Miss], [FA vs. CR], [FA vs. Miss], and [CR vs. Miss]. The anatomic atlases created by Duvernoy (1999) and Mai et al. (2008) were used to localize and describe activated regions. Voxels were reported in terms of the MNI coordinate space. Following neurological convention, the right side of an image corresponds to the right side of the brain. We kept uncorrected values at $p<0.005$ and used an extent threshold superior or equal to 5 adjacent activated voxels. In regions with strong, 
a priori hypothesis, such as the hippocampus and the parahippocampal area, the level of significance was tested with a $p<0.05$, corrected at the voxel level for multiple comparisons. Spheres of 10 -mm diameter were then used to define small volumes-of-interest (VOIs; small-volume correction, SVC; Worsley et al., 1996).

Specific analyses were performed on brain regions known to play a role in olfactory and memory processing. Since no probabilistic maps have been proposed for olfactory regions, anatomical VOIs in the piriform cortex, amygdala, hippocampus, and perirhinal and parahippocampal cortices were drawn from the MNI template, using the MRIcro ${ }^{1}$ and human brain atlases (Duvernoy, 1999; Mai et al., 2008). They were drawn from coronal slices for the piriform cortex (from $y=12.5$ anterior to -2.7 posterior to the anterior commissure), the amygdala (from $y=0$ to -13.3 ), the parahippocampal cortex (from $y=10$ to -45 ), and the perirhinal cortex (from $y=10$ to -12.5 ) and from sagittal slices for the hippocampus (from $x=-24$ to -38 on the left and $x=24$ to 38 on the right). The piriform cortex was divided along the $y$-axis into two areas: the anterior area (spreading from 12.5 to $3-4 \mathrm{~mm}$ ) and the posterior area (spreading from 3-4 to $-2.7 \mathrm{~mm}$ ). Orbitofrontal VOIs were spheres of $10-\mathrm{mm}$ radius located at coordinates previously identified by Gottfried and Zald (2005) in the left and right hemispheres (-22 30-17 and 24 $33-12$ ) as being the secondary olfactory cortex. The betas of each voxel were extracted and averaged for each VOI using the MarsBar toolbox ${ }^{2}$ for each of the 38 participants and each response category. ANOVA and mean comparisons were then performed to compare the levels of activation as a function of the groups (young and elderly) and response category (Hit, Miss, CR, FA).

Multivariate analyses of covariance and CVA were used to investigate functional relationships (i.e., connectivity and distance) between the major regional foci (Friston et al., 1996). The effects from activations, confounding effects, and error effects were assessed both in terms of single-voxel effects and of interactions among voxels. While MANCOVA allows for the inference that an entire network is engaged by tasks, the task effects were later characterized by CVA in terms of canonical vectors that best capture the activation effects, relative to error. The beta-values for each region-of-interest (anatomical VOIs and spheres of 10-mm radius located at the level of clusters for which significant activation differences were observed between Hits, Misses, CR, and FA) were calculated for each subject. Applying MANCOVA to these data allowed measuring the functional relation between these regions of interest. This measure gives an information different of this derived from the comparisons of the mean values relative to the conditions (classical contrast analysis). When activation foci were spatially closely related (only separated by 1 or 2 voxels), we computed means of the activation data. Data in young and elderly subjects were separately analyzed.

To determine the neural networks specifically activated for each response category (Hit, Miss, $\mathrm{CR}$, and FA) as a function of memory $\left(d_{L}^{\prime}\right)$ and bias $\left(C_{L}\right)$ participant scores, whole-brain linear regression analyses of beta-values were performed. The aim was to extract neural networks dependent on the subjects' ability to memorize odors (high or low scores) or on the decision rule they adopted when uncertain (liberal if they say "yes" or conservative if they say "no").

${ }^{1}$ http://www.mricro.com

${ }^{2} \mathrm{http}: / /$ marsbar.sourceforge.net
Since brain activation might depend on the time needed by the subjects to do the odor recognition task, we performed wholebrain linear regression analyses to detect neural networks for which activation was function of response time (RT) spent by subjects as a function of the four response categories. Finally, we examined whether neural networks activated during these different response could be also influenced by changes in inspiratory volumes, using linear regression analyses.

\section{RESULTS \\ BEHAVIORAL AND PHYSIOLOGICAL DATA}

The mean numbers of items classified as Hit and Miss (in percentage of targets), as well as FA and CR (in percent of distractors), were calculated for each group of subjects (young and elderly). The rate of no-response was $10.69 \%$ in young individuals and $20.36 \%$ in elderly subjects. A two-way ANOVA (Group $\times$ Response category) revealed a significant response category effect $\left[F_{(3,108)}=7.138\right.$; $p<0.001$ ] due to a greater number of correct responses (Hit and $\mathrm{CR}$, individually) than incorrect responses: FA ( $p$ 's $<0.005)$, and Miss ( $p$ 's $<0.003$; Figure 3). A nearly significant Group $\times$ Response category interaction $\left[F_{(3,108)}=2.470 ; p=0.066\right]$ could be partially explained by a number of correct responses (Hit and CR) tending to be significantly higher in young than in elderly subjects and a number of incorrect responses (FA and Miss) tending to be significantly higher in the elderly than in the young cohort ( $p$ 's $<0.001)$.

Response times were defined as the time interval between odorant delivery and the subject's response. Mean RTs in the encoding and retrieval sessions were $1.530 \pm 0.397$ and $2.934 \pm 0.858$ in young subjects and $1.634 \pm 0.638$ and $2.777 \pm 1.008$ in elderly subjects. For retrieval, mean RT was computed for the Hits, Misses, CR, and FA (Figure 2A). A two-way ANOVA (Group $\times$ Response category) demonstrated a significant effect in the response category $\left[F_{(3,108)}=5.858 ; p=0.001\right]$, mainly due to significantly higher RT in Miss responses than in FA $(p=0.004)$ and Hit $(p<0.001)$ responses, as well as a slightly significant Group $\times$ Response category interaction $\left[F_{(3,108)}=2.513 ; p=0.062\right]$. These data indicated that RT in
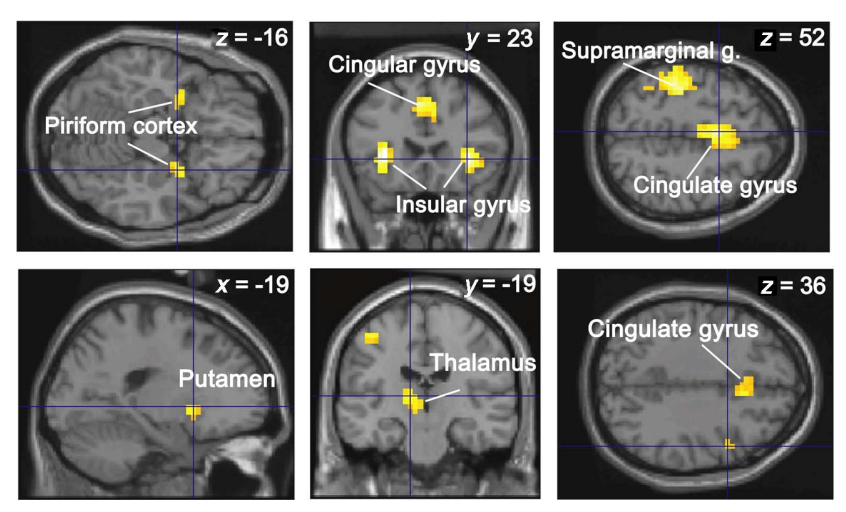

FIGURE 3 | Conjunction analysis between the four response categories and two groups. The activations are superimposed on sections of the normalized TI-weighted structural scan. The level of significance was set atp $<0.0001$ for display, uncorrected at the cluster level for multiple comparisons. 
young subjects tended to be higher in CR and Miss responses than in Hit ( $p=0.005$ and $p<0.0001$, respectively) responses and higher in Miss than FA $(p=0.006)$ responses.

Data calculated from the signal-detection theory showed that memory scores $d_{L}^{\prime}$ (Figure 2B) were significantly higher in young than elderly subjects $[0.915 \pm 0.638$ and $0.277 \pm 0.760$, respectively; $\left.F_{(1,36)}=7.452 ; p=0.010\right]$. No significant difference was found for bias scores $C_{L}[-0.087 \pm 0.503$ and $0.074 \pm 0.711$, respectively; $\left.F_{(1,36)}=0.601\right]$. However, differences in bias scores in subjects of both groups were negatively correlated with RT when they provided CR $(r=-0.440, p=0.006)$ and Miss responses $(r=-0.353, p=0.030)$. The more the subjects were conservative, the more time they needed to correctly or incorrectly reject the item was high. In other words, conservative subjects took more time to reject correctly new odorants or incorrectly old items.

The subjects were asked to breathe regularly and to avoid sniffing throughout the experiment, but variations in breathing amplitude were expected due to experimental conditions and the subjects' answers. Therefore, we analyzed a trial-specific breath parameter: the amplitude of the inspiration cycle following each odor stimulation. Mean cycle amplitudes were computed for the four response categories (Hit, Miss, CR, FA) in both groups of subjects. A two-way Group $\times$ Response category ANOVA revealed a significant effect for the response category factor $\left[F_{(3,108)}=3.243\right]$ : the inspiration volume was significantly greater for Miss than Hit responses $(p=0.049)$ and FA $(p=0.004)$, as well as for CR than FA responses $(p=0.018)$. These differences may be related to the higher RT observed in subjects when they rejected the item as new (Miss and CR), but no significant correlations between inspiratory volumes and RT were found $(p>0.05)$. No significant effects for Group factor $\left[F_{(1,108)}=1.447\right]$ and for Group $\times$ Response category interaction $\left[F_{(3,108)}=0.229\right]$ were found. Thus, our results indicate that the inspiratory volume was lower for recognition than for rejection, highlighting a close interaction between physiological response and cognitive processes, independently of RT.

\section{NEUROIMAGING DATA \\ Conjunction analysis}

Our first aim was to identify the common neural network activated for the four response categories (Hit, Miss, CR, FA) and the two subject groups. Conjunction analysis revealed significant regional cerebral blood flow increases in the piriform cortex, putamen, thalamus, insular, cingulate, inferior frontal, and supramarginal gyri (Figure 4 and Table 2).

\section{Contrast analyses}

The second study aim was to establish whether neural networks were differentially activated by response category by contrasting them twoby-two (Table 3 ). In young subjects, when we examined regions more activated for Hits, we found greater activation in the left hippocampus ([Hit-Miss] contrast) and the left parahippocampal gyrus ([Hit-FA] contrast; Figure 5). Examining the opposite contrasts ([FA-Hit], [CR-Hit], and [Miss-Hit]), we found that brain regions such as the cingulate, the middle and inferior frontal gyri, and the short insular and middle occipital gyri were less activated for Hit responses than for the other response categories. Interestingly, the right posterior piriform cortex was more activated in FA than Hits and Misses. In elderly subjects, the right hippocampus was more activated when subjects recognized the items as old (Hit and FA), regardless of accuracy, than when they rejected them (CR and Miss; Figure 4B).

Anatomical VOI analyses revealed significant activation differences in the right piriform and left perirhinal cortices as a function of experimental conditions and response categories (Figures 4C and 6). For the piriform cortex, we found a significant effect of Area $\left[F_{(1,108)}=24.831\right.$, $p<0.0001]$, indicating higher activation in the posterior than anterior area, and a significant Area $\times$ Response category $\times$ Group interaction $\left[F_{(3,108)}=3.082, p=0.0305\right]$. Mean comparisons revealed that activation was higher in FA than in Hit $(p=0.0145)$ and Miss $(p=0.0584)$ responses in young subjects, and also higher in Hit than Miss $(p=0.0111)$ responses and CR than Miss $(p=0.0256)$ responses in the posterior PC in elderly subjects. For the left perirhinal cortex, we observed a barely significant response category effect $\left[F_{(3,108)}=2.652\right.$, $p=0.0524$ ] due to a higher activation in both groups in FA and Miss than Hit responses ( $p=0.0124$ and $p=0.0488$, respectively).

We also compared activation patterns between groups for each response category (Table 4 and Figure 7 ). We mainly observed that the cuneus was less activated in elderly than young subjects, and the middle frontal gyrus was activated in elderly and deactivated in young subjects.

\section{MULTIVARIATE ANALYSES}

To characterize the brain responses in terms of functionally connected systems, we examined in young subjects the functional relationships between 22 structures identified by contrast analyses as a function of Hit, Miss, CR, and FA using multivariate analysis. MANCOVA revealed a significant overall effect of the four response categories $\left(\chi^{2}=206.97, T=7.12, p<0.05\right)$. The first three canonical variates resulting from a CVA respectively explained 23.8, 10.1, and $8.8 \%$ of the global variance. The expression of these variates over the four response categories and all subjects is plotted on Figure 8 to associate a "cognitive" meaning to these components. The first canonical variate (CV1) distinguished Hits and CRs from FAs and Misses, thus accounting for response accuracy (Figures 7A,C). CV2 mainly separated two categories of correct responses, as shown by the positive scores for Hits (0.193) vs. negative scores for CRs (0.212; Figure 7A). Finally, CV3 displays negative scores for FAs $(-0.175)$, clearly differing from positive scores for Misses (0.220).

To determine which portion of the network is explained by a specific $\mathrm{CV}$, each structures' loading on the components is plotted in Figures 7B,D. The functional linkage between two structures is revealed by the close location of their representative points on the eigenimages. In the two planes defined by the three CVs, we were able to distinguish three regions (outlined by the colored circles). The first CV (in red) corresponded to high values of CV1 and CV2. It includes the hippocampus $(-30-380)$ and the parahippocampal gyrus $(-30-34-12)$ and was clearly associated with Hits. The second CV (in yellow) includes the structures preferentially associated with FAs, such as the right posterior piriform cortex, the right middle frontal gyrus ( $42-848)$, and the right supramarginal gyrus ( $53-4240$ ). The third CV (in blue) is more associated with CRs than with the other response categories, and includes the right inferior frontal gyrus (42 2324 ), the cingulate gyrus (8 4 48, and $11-2752)$, and the left precentral gyrus $(-49-1936)$. Finally, the fourth CV (in green) includes areas more associated with Misses 


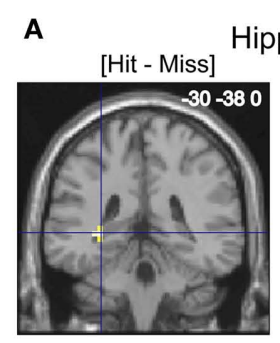

Hippocampus

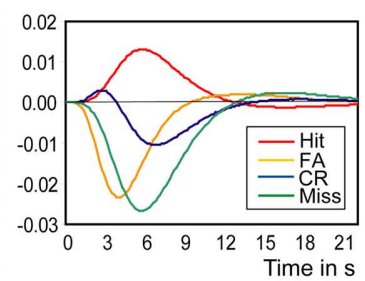

Cingulate gyrus
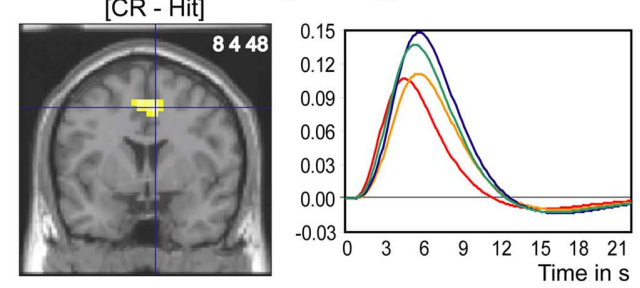

B

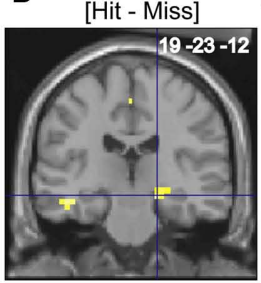

Hippocampus

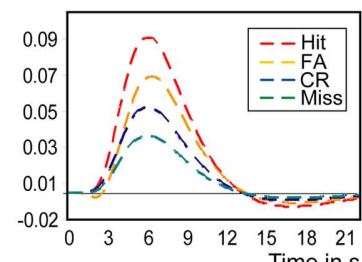

Middle Frontal gyrus

[Hit - Miss]
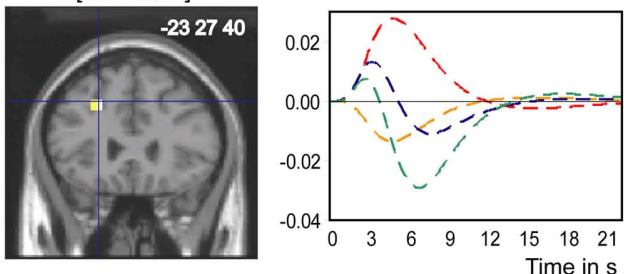

C

Right Posterior Piriform cortex
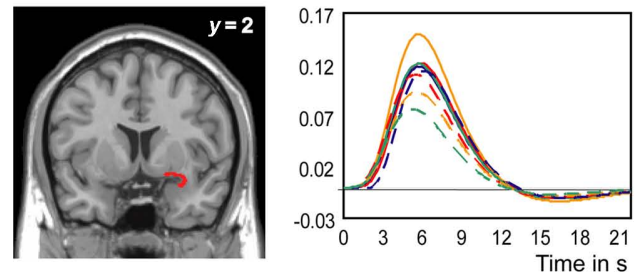

FIGURE 4 | Brain regions differentially involved in odor recognition memory as a function of the four response categories (Hit, Miss, CR, and FA). (A) In young subjects, the hippocampus, the parahippocampal gyrus, the cingulate gyrus, and the inferior frontal gyrus. (B) In elderly subjects, the hippocampus, the thalamus, the middle frontal gyrus, and the posterior cingulate gyrus. (C) In young and elderly subjects, the right posterior piriform

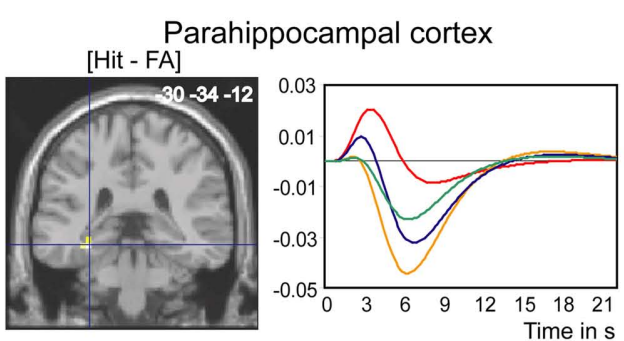

[Miss - Hit]
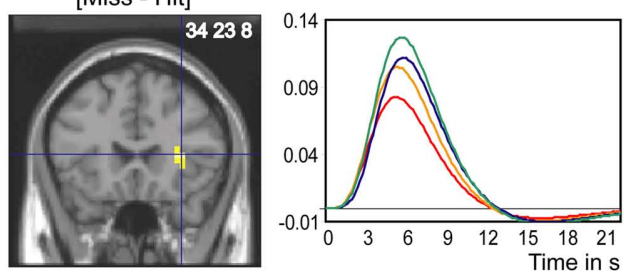

Thalamus
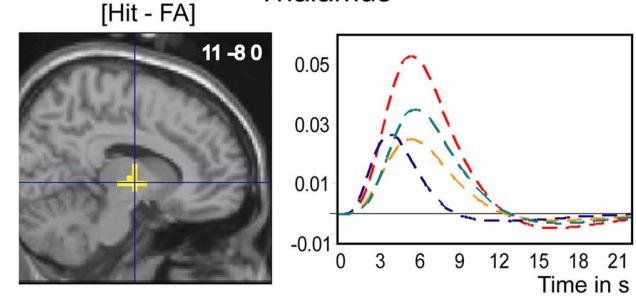

[Miss - FA]
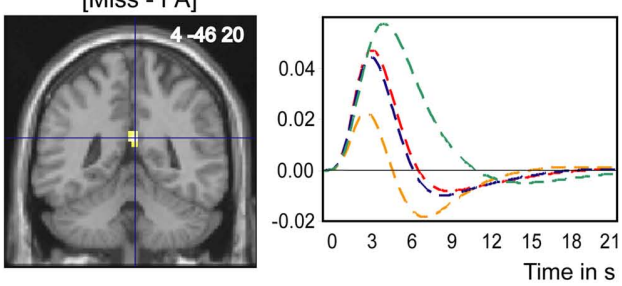

Left Perirhinal cortex
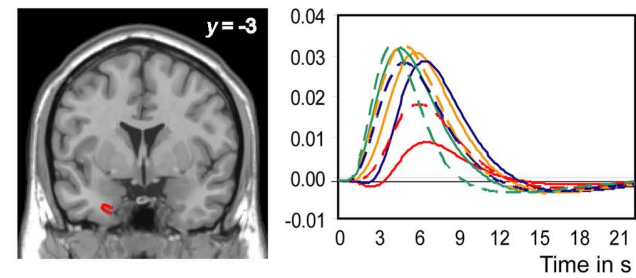

and left perirhinal cortices (VOIs). Contrasts are given between brackets. The clusters of activation and VOIs are superimposed on sections of the normalized TI-weighted structural scan. Graphs show the hemodynamic response functions using the canonical hrf and its time-derivative model in the four response categories during retrieval. The level of significance was set at $p<0.001$, uncorrected at the cluster level for multiple comparisons.

In elderly participants, the MANCOVA applied to data from the 22 structures identified in contrast analyses revealed a significant overall effect from the four response categories $\left(\chi^{2}=193.62\right.$, $T=6.45, p<0.05)$. The three CVs resulting from the CVA respectively explained $18.9,9.9$, and $8.2 \%$ of the global variance. The expression of these variates over all response categories and all than with the other response categories, such as the right insular gyrus (27 19 8), the anterior cingulate gyrus (CING: $-7.7-1.352$ ), and the middle temporal gyrus $(-42-380)$. The perirhinal cortex, the lateral orbital gyrus (38 27-4), the right inferior frontal gyrus (34 238 and 4919 4), the middle occipital gyrus (49-49 8), and the cerebellum $(42-49-32)$ were associated with Misses and FAs. 
Table 2 | Conjunction analysis between the four categories of response (Hit, Miss, CR, and FA) and the two groups (young and elderly) of subjects.

\begin{tabular}{lrrrrr}
\hline Cerebral areas & $\boldsymbol{k}$ & $\boldsymbol{T}$ & $\boldsymbol{x}$ & $\boldsymbol{y}$ & $\boldsymbol{z}$ \\
\hline Insular gyrus & 77 & 7.51 & 34 & 23 & 0 \\
Insular gyrus & 142 & 7.44 & -30 & 23 & 0 \\
Putamen & & 5.21 & -19 & 11 & 0 \\
Piriform cortex & & 4.71 & -23 & 4 & -12 \\
Piriform cortex & & 4.54 & -30 & 4 & -16 \\
Supramarginal gyrus & 123 & 6.48 & -42 & -30 & 52 \\
Cingulate gyrus & 145 & 6.43 & -4 & 4 & 52 \\
Cingulate gyrus & & 6.32 & -4 & 23 & 44 \\
Thalamus & 31 & 5.61 & -11 & -19 & 8 \\
Piriform cortex & 12 & 5.55 & 27 & 0 & -16 \\
Putamen & 19 & 4.92 & 15 & 11 & 0 \\
Inferior frontal gyrus & 6 & 4.27 & 49 & 8 & 36 \\
\hline
\end{tabular}

The level of significance was set at $p<0.0001$ for display, uncorrected at the cluster level for multiple comparisons, using small-volume correction, (Worsley et al., 1996). $k$, size of the cluster in number of connected voxels; T, Student's $t$ value; $x, y$, and $z, M N I$ coordinates (in $\mathrm{mm}$ ) of the maximum peak.

subjects are plotted in Figure 9. The CV1 axis separates Misses $(-0.272)$ from Hits (0.241; Figure 8A). The CV2 axis separates CRs and FAs from Hits and Misses (Figures 8A,C); positive scores for CRs (0.131) and FAs (0.168) differed from negative scores observed for the other responses (Hit: -0.166 ; and Miss: -0.133 ). Finally, the CV3 axis discriminates between FAs and CRs, as shown by the positive scores for FAs $(0.154)$ vs. negative scores for CRs $(-0.173)$.

The structures associated with the four response categories are depicted in Figures 8B,D. We primarily distinguish four regions. The first region (in yellow) corresponds to high values of CV2 and high values of CV3 and includes the hippocampus (19-23-12), the caudate nucleus ( -15274$)$, the cingulate gyrus ( $0-448)$, and the supramarginal gyrus $(61-4636)$. The second region (in red) includes the structures preferentially associated with Hits, such as the thalamus (THA1) and the middle occipital gyrus $(-42-80$ 8 ). The third region (in blue) is more associated with CRs and includes areas such as the inferior frontal gyrus (46 19 20), the middle temporal gyrus $(57-66.50)$, the cerebellum $(-4-76-12)$, and the putamen (230 4). Finally, the fourth region (in green) is preferentially associated with Misses and contains the cingulate gyrus (15 428$)$ and the precentral gyrus $(-42-1932)$. The angular gyri (-27 -57 40, $53-5740,-46-6132)$ are associated with Hits, as with FAs and Misses. The perirhinal cortex VOI is associated with FAs and CRs.

\section{Linear regression analyses}

The third aim of this study was to examine activation patterns with regard to individual differences in memory score $\left(d_{L}^{\prime}\right)$ and response bias score $\left(C_{L}\right)$ using linear regression analyses (Tables 5 and 6). Although neither score was significantly correlated $(p=0.182)$, we chose not to perform multiple regression analyses to avoid masking any shared effect. The analyses revealed that memory scores were negatively correlated with the activation (beta-values) of different regions in the medial temporal lobe for specific response categories. In particular, activation in the anterior hippocampus $(30-11-12$ and $-30-8-16$ ) was associated with accurate responses (Hit and $\mathrm{CR}$, respectively; Figure 9A), and the middle frontal gyrus was associated with Hits, CRs, and FAs in both groups. Only two activation spots were positively correlated with memory scores when the subjects provided true recognition: the right area piriformis insulae and the insular gyrus. Nevertheless, a thorough examination of data revealed that activation of the first region was mainly from elderly subjects. Considering the other response categories (Miss, CR, FA), activation of only a few regions was positively correlated with memory scores in elderly participants, but no region had an activation positively correlated in young participants.

Response bias scores were positively correlated with activation of different medial temporal lobe regions for specific response categories. Thus, the right anterior hippocampus $(30-15-16$ and 27 $-11-20$ ) activated when subjects recognized an item as old (Hit and FA, respectively; Figure 9B). However, a detailed examination of the two groups revealed that these effects were due to elderly subjects $(27-11-16, k=18, T=6.83, p=0.014)$.

Since activation might depend on the RT, we tested for this effect during Hit, Miss, FA, or CR. RTs were negatively correlated with activation level during FA in the cerebellum $(-8-87-28: k=5$, $\left.T=4.25, p_{\text {uncor }}=0.055\right)$, the putamen $(-23-118: k=8, T=4.13$, $\left.p_{\text {uncor }}=0.019\right)$, and during Miss in the cerebellum $(15-34-16$ : $\left.k=6, T=3.76, p_{\text {uncor }}=0.041\right)$. They were positively correlated with activation level during $\mathrm{CR}$ in the precuneus and superior occipital gyrus $\left(-15-6124\right.$ and $\left.-15-9516: k=106, T=5.26, p_{\text {uncor }}<0.001\right)$.

As we observed variations in inspiratory volumes as a function of categories of responses, we finally examined whether activation could be influenced by these physiological changes. The level of activation was negatively correlated with inspiratory volumes in the cerebellum $\left(0-53-4: k=7, T=5.03, p_{\text {uncor }}=0.026\right)$ and the thalamus $\left(0-53-4: k=7, T=5.03, p_{\text {uncor }}=0.026\right)$ during FA, and positively correlated with activation in the trunk $(0-15-12: k=6$, $T=3.79, p_{\text {uncor }}=0.041$ ) during Miss.

\section{DISCUSSION}

The aim of the present study was to identify the specific neural correlates of olfactory recognition memory. Specifically, we sought to elucidate the common and disparate neural networks underlying tasks performance in true and false memory, as well as correct and incorrect no-recognition of odors. In addition to a common network activated in all four response categories, we found greater activation in the hippocampus and the parahippocampal gyrus for true memories in young subjects than the other response categories, and false recognition involved a larger network, including the perirhinal cortex, respectively highlighting roles in recollection and familiarity processes. In elderly individuals, the hippocampus was equally activated in both true and false memories, and activation patterns were less clearly segregated than in young adults. We also found that activation in the hippocampus was negatively correlated with memory scores but positively correlated with bias scores.

\section{A NEURAL NETWORK COMMON TO ALL CATEGORIES OF MEMORY RESPONSE}

The neural network activated in the four response categories (Hit, Miss, CR, FA) included regions involved in olfactory and memory processes. Regions involved in olfactory perception consist of the piriform cortex, the insular gyrus, the cingulate gyrus, and the 
Table 3 | Brain areas activated as a function of contrasts between response categories.

\begin{tabular}{|c|c|c|c|c|c|c|c|c|}
\hline Group & & Contrasts & Brain areas & $\boldsymbol{k}$ & $T$ & $x$ & $y$ & $z$ \\
\hline \multirow[t]{19}{*}{ Young } & \multirow[t]{2}{*}{ Hit- } & Miss & Hippocampus* & 9 & 3.91 & -30 & -38 & 0 \\
\hline & & FA & Parahippocampal gyrus* & 4 & 4.40 & -30 & -34 & -12 \\
\hline & \multirow{8}{*}{ Miss- } & \multirow{6}{*}{ Hit } & Middle frontal gyrus* & 12 & 4.27 & -23 & 46 & 24 \\
\hline & & & Inferior frontal gyrus* & 9 & 4.04 & 49 & 19 & 4 \\
\hline & & & Inferior F. g., pars triangu & 28 & 3.97 & 34 & 23 & 8 \\
\hline & & & Middle occipital gyrus & 10 & 3.57 & 49 & -49 & 8 \\
\hline & & & Cingulate gyrus* & 7 & 3.40 & 8 & 46 & 24 \\
\hline & & & Cingulate gyrus & 12 & 3.47 & 0 & -42 & 56 \\
\hline & & \multirow[t]{2}{*}{ FA } & Insular gyrus* & 12 & 4.76 & 27 & 19 & 8 \\
\hline & & & Cingular gyrus* & 8 & 4.20 & -11 & -4 & 52 \\
\hline & \multirow{6}{*}{$\mathrm{CR}-$} & \multirow{3}{*}{ Hit } & Cingulate gyrus & 25 & 4.25 & 8 & 4 & 48 \\
\hline & & & Cingulate gyrus & & 3.56 & -4 & 4 & 52 \\
\hline & & & Cerebellum & 8 & 3.52 & 42 & -49 & -32 \\
\hline & & \multirow[t]{3}{*}{ Miss } & Inferior frontal gyrus* & 9 & 4.31 & 42 & 23 & 24 \\
\hline & & & Precentral gyrus & 10 & 3.91 & -49 & -19 & 36 \\
\hline & & & Cerebellum & 8 & 3.75 & 38 & -42 & -28 \\
\hline & \multirow[t]{2}{*}{ FA- } & Hit & Middle frontal gyrus* & 7 & 3.52 & 42 & -8 & 48 \\
\hline & & $\mathrm{CR}$ & Supramarginal/angular g.* & 12 & 4.21 & 53 & -42 & 40 \\
\hline & \multirow[t]{10}{*}{ Hit- } & \multirow[t]{3}{*}{ Miss } & Middle frontal gyrus* & 14 & 3.93 & -23 & 27 & 40 \\
\hline \multirow[t]{19}{*}{ Elderly } & & & Cingulate gyrus* & 7 & 3.62 & 4 & -27 & 56 \\
\hline & & & Cingulate gyrus & 9 & 3.58 & 0 & -4 & 48 \\
\hline & & \multirow[t]{7}{*}{ FA } & Cingulate gyrus & 10 & 4.33 & 4 & -8 & 52 \\
\hline & & & Thalamus & 22 & 3.90 & 15 & -8 & -4 \\
\hline & & & Thalamus & & 3.61 & 11 & 0 & 0 \\
\hline & & & Putamen & & 3.29 & 23 & 0 & 4 \\
\hline & & & Cerebellum & 8 & 3.69 & -4 & -76 & -12 \\
\hline & & & Middle temporal gyrus & 11 & 3.62 & 57 & -65 & 0 \\
\hline & & & Superior lingual gyrus & 11 & 3.36 & 11 & -72 & -4 \\
\hline & \multirow[t]{8}{*}{ Miss- } & \multirow[t]{2}{*}{ Hit } & Precentral gyrus & 10 & 3.80 & -42 & -19 & 32 \\
\hline & & & Cuneus/gyrus descendens & 7 & 3.40 & 23 & -91 & -4 \\
\hline & & \multirow[t]{3}{*}{$\mathrm{CR}$} & Angular gyrus & 9 & 4.21 & -46 & -61 & 32 \\
\hline & & & Cingulate gyrus* & 9 & 3.73 & 15 & 4 & 28 \\
\hline & & & Angular gyrus & 9 & 3.53 & -27 & -57 & 40 \\
\hline & & \multirow[t]{3}{*}{ FA } & Inferior lingual gyrus & 11 & 3.56 & -4 & -80 & -12 \\
\hline & & & Cingulate gyrus* & 8 & 3.48 & 4 & -46 & 20 \\
\hline & & & Middle temporal gyrus & 8 & 3.32 & 57 & -68 & 0 \\
\hline & CR- & Hit & Inferior frontal gyrus* & 9 & 3.72 & 46 & 19 & 20 \\
\hline & FA- & $\mathrm{CR}$ & Head of caudate nucleus & 12 & 3.64 & -15 & 27 & 4 \\
\hline
\end{tabular}

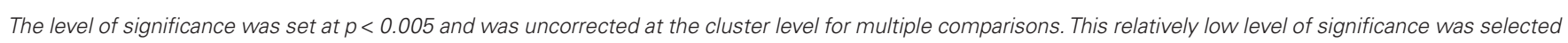
because differences between cognitive processes associated with different response categories are likely to be relatively small (Heun et al., 2004; Kim and Cabeza,

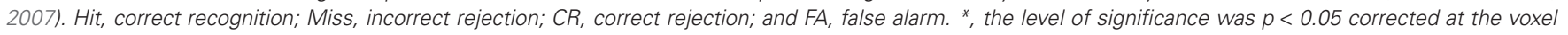
level for multiple comparisons, using small-volume correction (Worsley et al., 1996). Other abbreviations: see Table 2. 


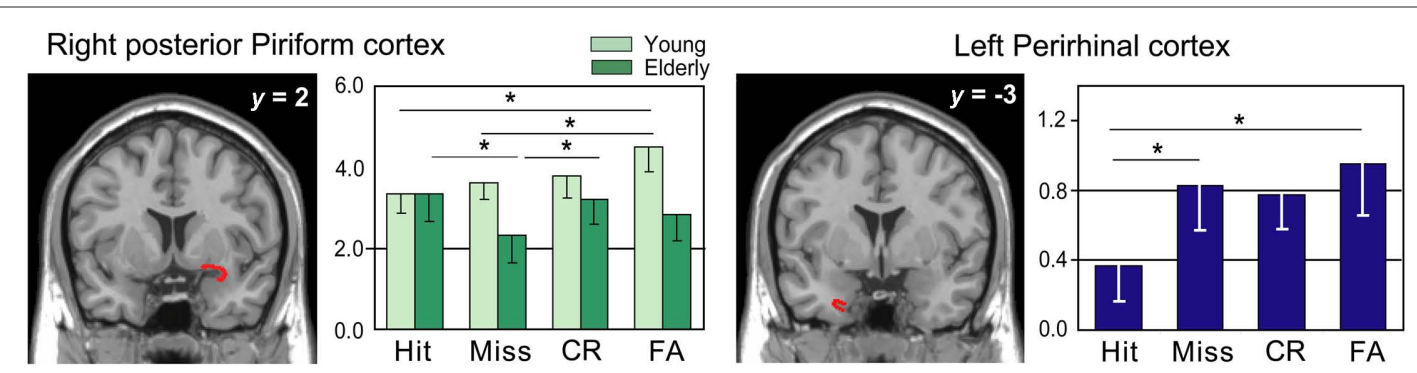

FIGURE 5 | rCBF differences found in the right piriform cortex and the left perirhinal cortex. The statistical parametric mapping (SPMs) are superimposed on coronal sections of a Tl-weighted scan. Graphs: level of activations for Hits, Misses, CR, and FA in young and elderly subjects in the right piriform cortex and in both groups in the left perirhinal cortex. Vertical bars, standard errors of the mean; ${ }^{*} p<0.05$.
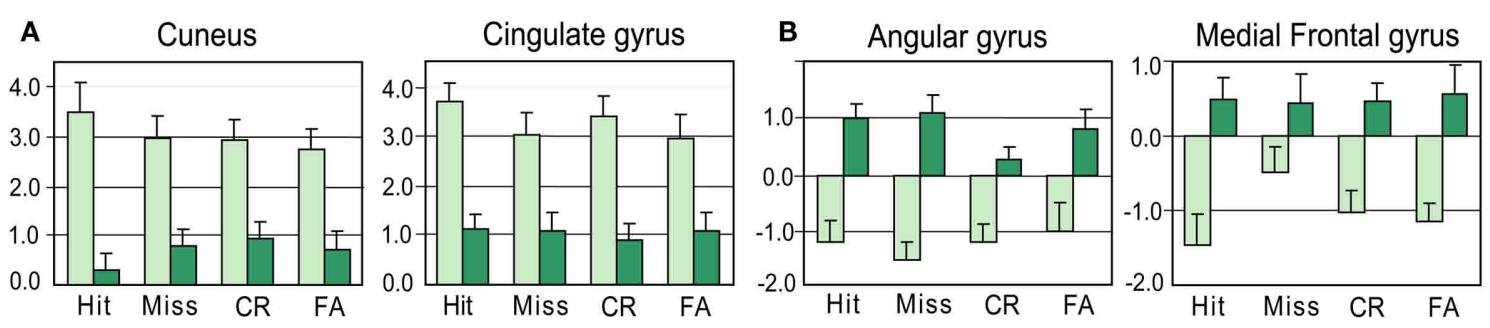

FIGURE 6 | Levels of activation for Hits, Misses, CR, and FA in young and elderly. (A) In the cuneus (11 -87 4) and the cingulate gyrus (4 -30 28). (B) In the angular (-46 -61 36) and middle frontal (-34 3044$)$ gyri. Vertical bars, standard errors of the mean.

thalamus (Gottfried et al., 2002; Royet et al., 2003; Plailly et al., 2008). The supramarginal gyrus that belongs to the parietal lobe, has been implicated in most studies on recognition memory (Leube et al., 2003; Heun et al., 2004; Garoff-Eaton et al., 2006) and in long-term memory of odors (Cerf-Ducastel and Murphy, 2006).

\section{TRUE AND FALSE RECOGNITION}

Signal-detection-based models assume the placement of items on a familiarity continuum, such that old items fall on the high end of the continuum and new items fall on the low end of the continuum (Yonelinas et al., 1996; Wixted and Stretch, 2004). However, the familiarity of old and new items is normally distributed across the continuum, and items overlap each other. In such a model, FA can be considered a familiarity process, whereas Hit can be considered a recollection process. When contrasting activation images obtained for true (Hit) and false (FA) recognition in young subjects, we found greater activation in the hippocampus and the parahippocampal gyrus when recognition was accurate and greater activation in the perirhinal cortex when recognition and rejection were inaccurate (FA and Miss). Although we used a yes-no-recognition task and did not rate familiarity and recollection via a remember-know procedure, our results are consistent with evidence from neuropsychological and neuroimaging studies, suggesting a specific role for the hippocampus and the parahippocampal gyrus in recollection that requires greater recovery of sensory or contextual information but is not sensitive to differences in item familiarity, and for the perirhinal cortex, which is consistently correlated with familiarity (Yonelinas et al., 2005; Daselaar et al., 2006; Montaldi et al., 2006; Eichenbaum et al., 2007). In addition to the perirhinal cortex, several areas were preferentially associated with false recognition, such as the right posterior piriform cortex, the posterior cingulate gyri, the middle frontal gyrus, and the supramarginal gyrus (Figures 7A,B). In the past, we have shown a relationship between activation in the piriform cortex and the judgment of odor familiarity (Plailly et al., 2007). Here, although the piriform cortex was activated in the four categories of memory response, the right posterior piriform cortex appeared to be more activated for FA, reinforcing the role of this region in the familiarity judgment of odors. Thus, recent studies indicate that categorical perception and odor discrimination are encoded in this region (Gottfried, 2010), and activation in this region during FA may reflect the familiarity sensation related to the difficulty of discriminating items. Activation in the anterior and dorsolateral prefrontal cortex and the posterior cingulate has previously been associated with familiarity (Yonelinas et al., 2005; Daselaar et al., 2006), and patients with dorsolateral prefrontal lesions are significantly impaired in familiarity (Duarte et al., 2005).

\section{CORRECT AND INCORRECT REJECTION}

Very few studies have examined the brain activity associated with the rejection process (Goldmann et al., 2003; Heun et al., 2004; Slotnick and Schacter, 2004). Here, when young participants correctly rejected odors, it was noteworthy to find several spots of activation in the cingulate gyrus (Table 3), which was also associated, but less clearly, with incorrect rejection. Previous studies document activation within the cingulate when subjects are confident in their response (Chua et al., 2006; Moritz et al., 2006; Kim and Cabeza, 2007). It has been reported that conviction facilitates decisive actions, whereas doubt cautions a subject to withhold a response and to prolong the search process (Moritz et al., 2006). In our study, it is possible that subjects compared a new item with all items previously smelled prior to correctly or 
Table 4 | Differences of activation between the young and elderly for Hit, Miss, false alarm (FA), and correct rejection (CR).

\begin{tabular}{|c|c|c|c|c|c|c|c|}
\hline Contrast & Response category & Brain areas & $\boldsymbol{k}$ & $\boldsymbol{T}$ & $x$ & $y$ & $z$ \\
\hline \multirow[t]{19}{*}{ Young-elderly } & \multirow[t]{4}{*}{ Hit } & Cingulate gyrus & 16 & 5.65 & 4 & -30 & 28 \\
\hline & & Cuneus & 52 & 5.32 & 11 & -87 & 4 \\
\hline & & Insular gyrus & 7 & 4.25 & -38 & 4 & -12 \\
\hline & & Head of caudate nucleus & 8 & 4.03 & 11 & 15 & -4 \\
\hline & \multirow{5}{*}{ Miss } & Insular gyrus & 7 & 4.52 & -38 & 4 & -16 \\
\hline & & Cuneus & 16 & 4.47 & -15 & -76 & 8 \\
\hline & & Cingulate gyrus & 28 & 4.27 & 4 & 15 & 44 \\
\hline & & Cingulate gyrus & 16 & 4.12 & 0 & -23 & 28 \\
\hline & & Cuneus & 9 & 3.98 & 15 & -80 & 8 \\
\hline & \multirow{5}{*}{$\mathrm{CR}$} & Inferior occipital gyrus & 8 & 4.38 & -30 & -95 & -12 \\
\hline & & Cuneus & 29 & 4.35 & 4 & -87 & 0 \\
\hline & & Cuneus & 6 & 4.33 & 15 & -76 & 8 \\
\hline & & Fusiform gyrus & 10 & 4.12 & 38 & -42 & -24 \\
\hline & & Insular gyrus & 9 & 3.96 & -38 & -11 & 16 \\
\hline & \multirow[t]{5}{*}{ FA } & Cingulate gyrus & 7 & 4.52 & 0 & -30 & 24 \\
\hline & & Cuneus & 9 & 4.48 & -15 & -84 & 8 \\
\hline & & Cuneus & 20 & 4.34 & 11 & -87 & 12 \\
\hline & & Cuneus & & 4.08 & -4 & -76 & 8 \\
\hline & & Insular gyrus & 15 & 4.22 & 46 & 11 & -4 \\
\hline \multirow{6}{*}{ Elderly-young } & Miss & Angular gyrus & 9 & 4.43 & 57 & -65 & 24 \\
\hline & \multirow[t]{2}{*}{$\mathrm{CR}$} & Angular gyrus & 14 & 5.79 & -46 & -61 & 44 \\
\hline & & Angular gyrus & 5 & 3.59 & 46 & -76 & 32 \\
\hline & \multirow[t]{3}{*}{ FA } & Angular gyrus & 12 & 5.76 & -38 & -72 & 32 \\
\hline & & Supramarginal gyrus & 8 & 4.66 & -42 & -57 & 28 \\
\hline & & Lingual or fusiform gyrus & 8 & 4.36 & -30 & -42 & -8 \\
\hline
\end{tabular}

The level of significance was set at $p<0.001$ uncorrected at the cluster level for multiple comparisons. CC, corpus callosum. Other abbreviations: see Table 2.

incorrectly rejecting it, a search necessitating additional time that is concordant with our behavioral results. Nevertheless, this exploratory activity during rejection does not necessarily reflect doubt but may be associated with confidence judgments. Thus, Moritz et al. (2006) also found that increased confidence is associated with activation in the cingulate gyrus for the four memory response categories, the neuronal modulation of confidence then following a stepwise activation pattern, that is activation for high-confident responses and de-activation for low-confident responses.

When subjects incorrectly rejected odors, a wide neural network, including the right insula and precuneus, and bilateral prefrontal cortex, was activated in addition to the cingulate gyrus. Insular and prefrontal regions are more active during the engagement of effortful post-retrieval processes (Goldmann et al., 2003). The precuneus, that is part of the superior parietal lobe, is active when the need for top-down attention to memory retrieval is maximal (e.g., for familiar vs. recollected memories or memories retrieved with low vs. high confidence; Ciaramelli et al., 2008).

\section{MEMORY AND BIAS SCORES}

The influence of memory scores and bias on brain activation was examined in both groups of participants. We observed that activation in the anterior hippocampus was inversely correlated with memory scores when subjects' answers were Hits and CR: 

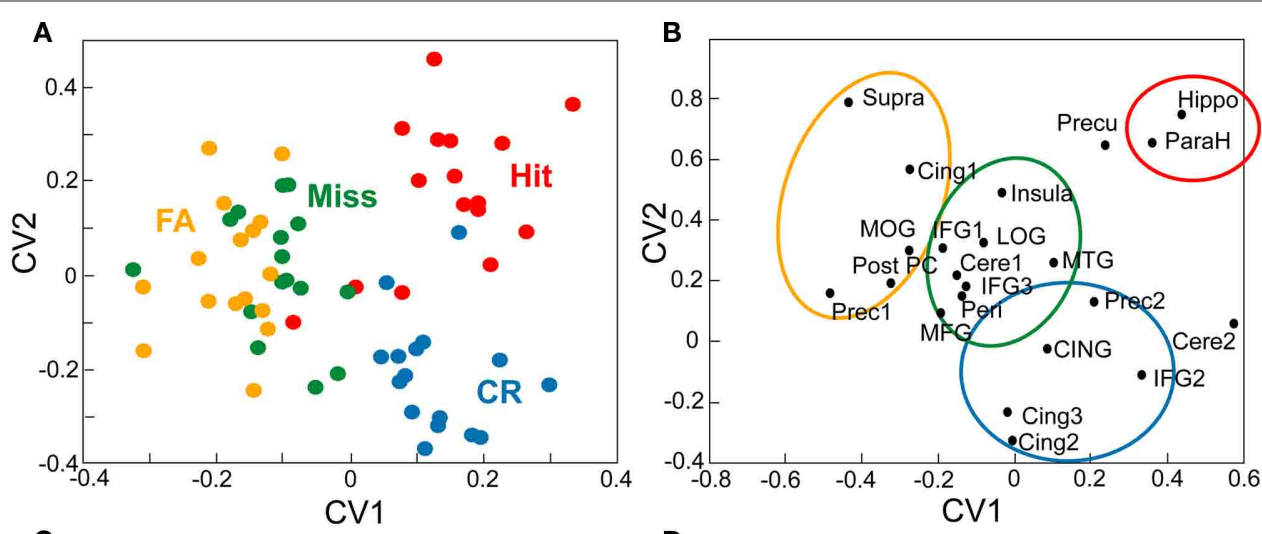

\section{C}

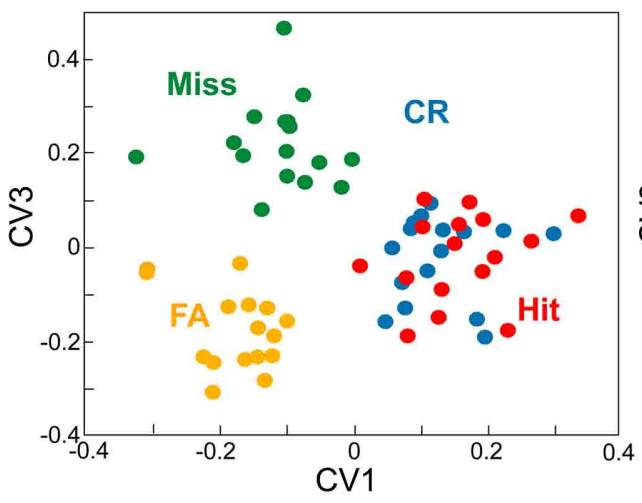

D

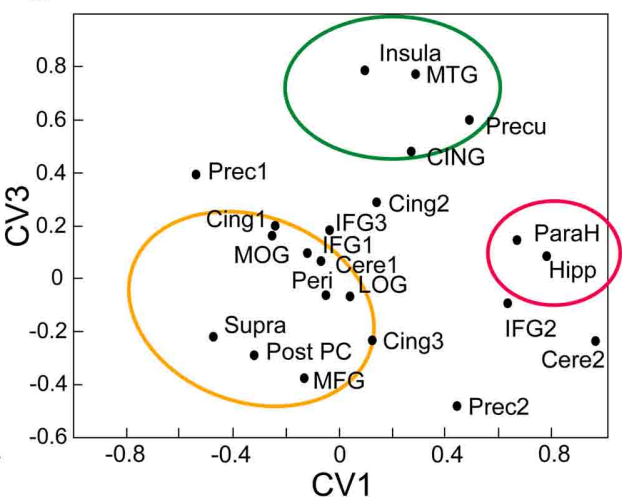

Cere $142-49-32$

Cere2 $38-42-28$

CING $-7.7-1.352$

Cing1 $0-4256$

Cing2 8448

Cing3 $11-2752$

Hipp -30 -38 0

IFG1 34238

IFG2 422324

IFG3 49194

Insula 27198

LOG $3827-4$

MFG 42 -8 48

MOG $49-498$

MTG $-42-380$

ParaH -30 -34 -12

Peri Left

Post PC Right

$\begin{array}{llll}\text { Prec1 } & -46 & -11 & 40\end{array}$

Prec2 $-49-1936$

Precu $11-5736$

Supra $53-4240$
FIGURE 7 | Canonical variate analysis of activations recorded in 22 structures in $\mathbf{1 6}$ young subjects. (A-C) Expression of canonical variates over all response categories (Hit, Miss, CR, and FA) and all subjects (one point per subject). (B-D) Loading of each structure on the canonical variates. (A,B) planes defined by the canonical variates 1 and 2 (CV1 and CV2). (C,D) planes defined by the canonical variates 1 and 3 (CV1 and CV3).
Cere, cerebellum; CING, mean of three ROls in cingulate; Cing. cingulate; Hipp, hippocampus* IFG, inferior frontal gyrus, LOG, lateral occipital gyrus; MFG, middle frontal gyrus; MOG. middle occipital gyrus; MTG. middle temporal gyrus; ParaH, parahippocampal; reri, perihippocampal; Post PC; posterior piriform cortex; Prec, precentral; Precu, precuneus; Supra, supramarginal. the higher the memory scores, the more the activation decreased. This surprising result is reminiscent of a recent finding in which we demonstrated that the activity in the hippocampus (but also the piriform and orbitofrontal cortices) is negatively correlated with experience during the creation of an odor mental image in professional perfumers (Plailly et al., 2011). Here, we may hypothesize that greater ease in correctly recognizing old odors (Hit) and rejecting new items (CR) results in less activation in the hippocampus. This process was not related to RT because no significant correlation between activation and RT was found. Interestingly enough, Stark and Squire (2001) found that activity in the medial temporal lobe is higher during quiet rest periods than during mind-numbing tasks, such as deciding whether or not numbers are odd or even, suggesting that periods of rest are associated with significant cognitive activity. These findings further lead to the view that the level of hippocampal activity is not necessarily a sign of the strength of a specific memory task but can on the contrary mirror the difficulty in remembering events.

Another striking finding observed in our study was poverty of regions positively correlated with memory scores when young subjects responded with true recognition. According to the contemporary models of memory formation, experience is initially encoded in hippocampal and cortical networks in parallel, and subsequent reactivation of the hippocampal network afterward reinstates activity in different cortical networks (Frankland and Bontempi, 2005). Here, the disengagement of the hippocampus may be associated with activation in the right insula, an area that pertains to the secondary olfactory cortex. On the one hand, it is possible that other regions involved were undetected by fMRI due to broad network distribution. On the other hand, if this mechanism of memory reactivation is conceivable after a high-demand cognitive learning task or as a function of experience (Plailly et al., 2011). It is less easily conceivable in the frame of a simple yesno-recognition memory task. Other alternative explanations are then required.

Concerning response bias, we found that activity in the anterior hippocampus was positively correlated with bias scores for true and false recognition, though positive correlation in the hippocampus for FA was primarily established from elderly subjects. The more conservative the subject's, the more the anterior hippocampus was activated. Thus, we can hypothesize that conservative subjects produced more exploratory effort and consequently displayed increased activation in this area, a result that strongly contrasts with the decrease in activation observed in subjects with high memory 

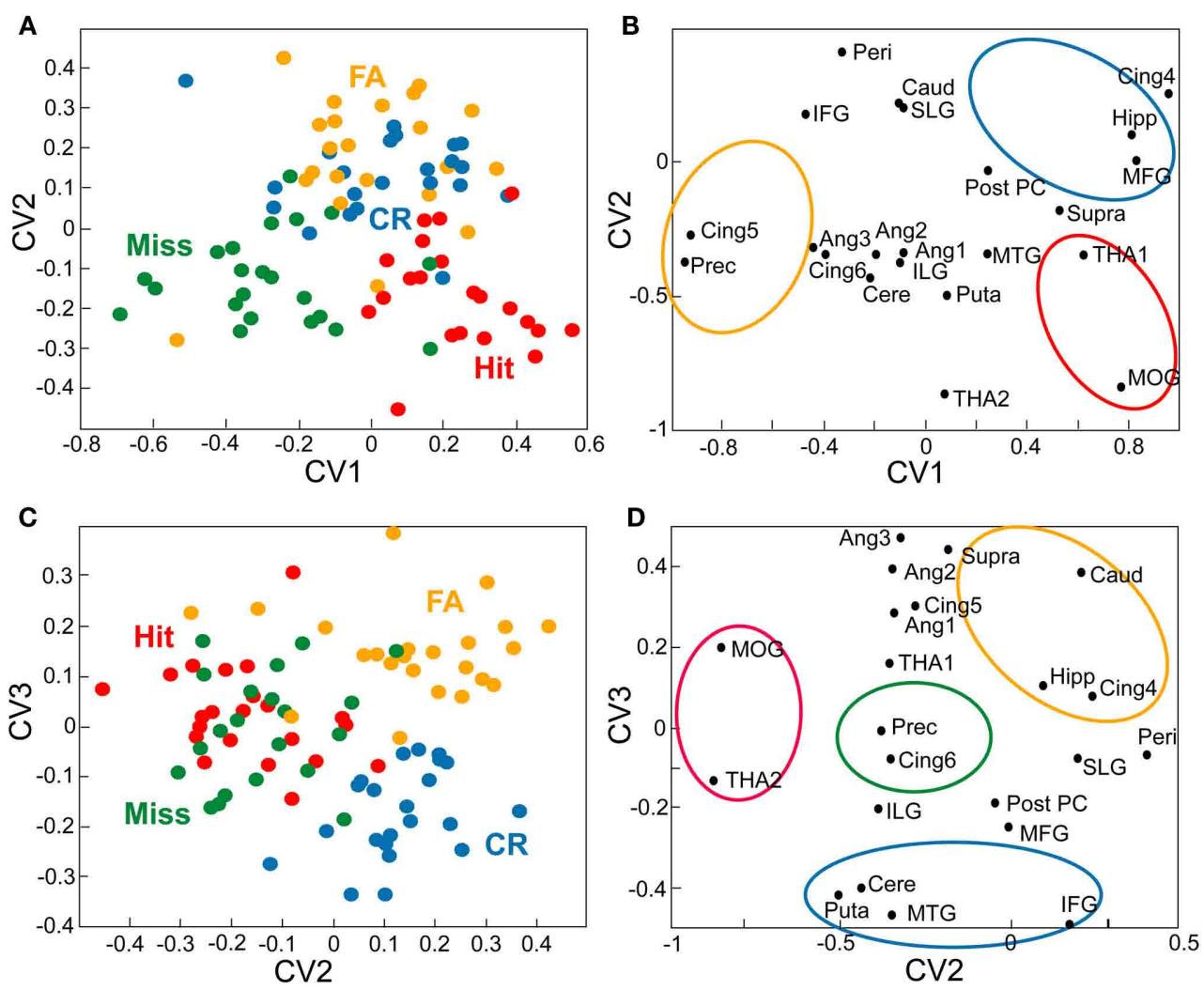

Ang1 $53-5740$

Ang2 -46 -61 32

Ang3 $-27-5740$

Caud -15 274

Cere $-4-76-12$

Cing $4 \quad 0-4 \quad 48$

Cing5 15428

Cing6 $4-4620$

Hipp1 $27-19-16$

IFG 461920

ILG $\quad-4-80-12$

MFG -23 2740

MOG -42 -80 8

MTG $57-66.50$

Peri Left

Post PC Right

Prec -42 -19 32

Puta 2304

SLG $11-72-4$

Supra $-61-46 \quad 36$

THA1 12.3 -8 2.7

THA2 1500

FIGURE 8 | Canonical variate analysis of activations recorded in 22 structures in 22 elderly subjects. (A,B) Planes defined by CV1 and CV2. (C,D) Planes defined by CV2 and CV3. Ang, angular; Caud, caudate; ILG, inferior lingual gyrus; Puta, putamen; SLG, superior lingual gyrus; THA. Mean of two or three ROIs in the thalamus. See Figure 7 for details and other abbreviations.

Table 5 | Cerebral areas where activation was positively or negatively correlated with memory scores for Hits, Misses, correct rejections (CR) and false alarms (FA).

\begin{tabular}{|c|c|c|c|c|c|c|c|}
\hline Response category & Correlation & Brain area & $\boldsymbol{k}$ & $T$ & $\boldsymbol{x}$ & $y$ & $z$ \\
\hline \multirow[t]{4}{*}{ Hit } & Positive & Piriform/area piriformis insulae/insular gyrus* & 15 & 4.30 & 46 & 11 & -16 \\
\hline & & Insular gyrus* & 16 & 4.13 & 46 & -4 & 0 \\
\hline & & Middle frontal gyrus & 7 & -4.27 & 34 & 23 & 48 \\
\hline & & Hippocampus* & 4 & -3.85 & 30 & -11 & -12 \\
\hline \multirow[t]{4}{*}{ FA } & Negative & Middle frontal gyrus* & 16 & -4.73 & 46 & 27 & 36 \\
\hline & & Angular gyrus & 12 & -4.31 & 53 & -57 & 40 \\
\hline & & Middle temporal gyrus & 5 & -4.11 & 57 & -46 & 0 \\
\hline & & Middle frontal gyrus & 13 & -4.03 & -42 & 23 & 40 \\
\hline
\end{tabular}

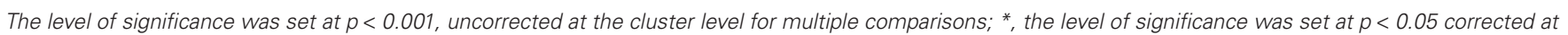
the voxel level for multiple comparisons, using small-volume correction (Worsley et al., 1996). Abbreviations: see Table 2.

scores and correct recognition of odors. Although participants did not explicitly rate confidence in judgments, a conservative attitude involves that subjects answer in the affirmative only when they are sure in their response. Our results are then consistent with recently published data (Wais et al., 2010) indicating that hippocampal activity is readily detectable when confidence in memory is strong.

\section{EFFECT OF AGING}

The current neuroimaging data reveal similarities between young and elderly subjects. For example, activation of the anterior hippocampus was negatively associated with memory scores in both elderly and young individuals. However, several differences between groups were also noted. First, activation in the hippocampus was 

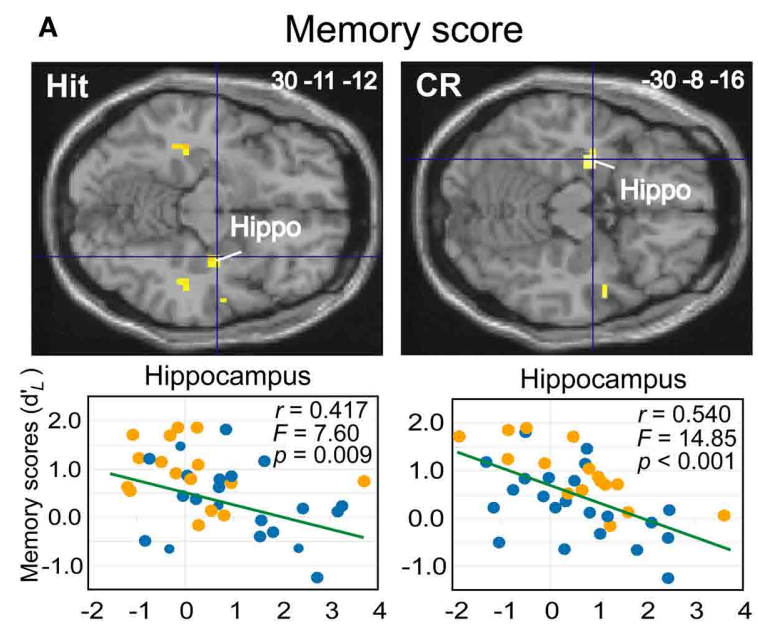

FIGURE 9 | Linear regression analyses. (A) Decreases of activation intensity (beta values) in the hippocampus as a function of memory scores $\left(d_{L}^{\prime}\right)$ for Hit and CR in all subjects. (B) Increases of activation intensity in the hippocampus as a function of response bias scores $\left(C_{L}\right)$ for Hit and FA in the elderly. The activations are superimposed on axial sections of the normalized TI-weighted structural
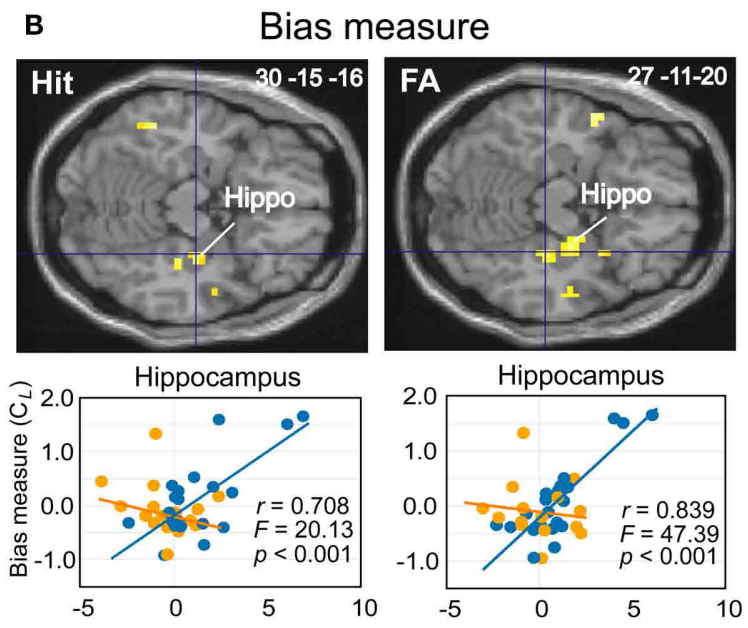

scan. Scatter diagrams represent variability in the levels of activation of young (orange dots) and elderly (blue dots) subjects for a type of response category as a function of memory or response bias scores. Green, blue, and orange lines, regression lines for all subjects, elderly and young, respectively. Hippo, hippocampus.

specific to accurate recognition (Hit) in young subjects, whereas this region was also activated with FA responses in elderly subjects. This result contrasts with studies revealing recollection deficits with reduction in hippocampus activity in elderly subjects (Cabeza et al., 2004), demonstrating that this area can also be activated when subjects are mistaken. Furthermore, this difference was even more notable when elderly subjects adopted a conservative attitude (Figure 9B), that is, said "yes" only when they were certain.

Second, in addition to activation within the hippocampus and the parahippocampal cortex, several other areas, including the thalamus and the middle frontal, cingulate and angular gyri, were significantly activated during odor recognition (Hit and FA) in elderly subjects. This finding is consistent with the previous literature (Cabeza et al., 2004; Dennis et al., 2008) and supports the view that elderly subjects compensate for declining processes with the recruitment of additional neuronal resources that are unnecessary for young adults. These data are also consistent with Montaldi et al. (2006)'s results demonstrating that as strength of familiarity increases, activity in the left dorsomedial thalamus, left ventrolateral and anteromedial frontal cortex, posterior cingulate gyrus and left parietal neocortex increase linearly. The transthalamic pathway is considered an active modulatory target of olfactory attention (Plailly et al., 2008), and the supramarginal/angular gyri activated here in elderly subjects and deactivated in young subjects are reported to play a key role in supporting recollection (Vilberg and Rugg, 2008). Recently, Quamme et al. (2010) demonstrated that this region proactively establishes an internally directed attentional state ("listening for recollection") that fosters recollection, selectively decreasing FAs to related lures. While elderly subjects displayed difficulty in recognizing odors, they may expend effort carefully listening for recollected details. Furthermore, in comparing activation patterns between both groups for each response category, we observed that the cuneus was less activated in elderly than in young subjects, and the middle frontal gyrus was activated in elderly subjects and deactivated in young individuals. The agerelated reduction in occipital activity coupled with increased frontal activity is consistent with the posterior-anterior shift in aging (PASA) model, which is also attributed to functional compensation (Davis et al., 2008).

These findings show that olfactory memory processes notably differ between young and elderly subjects. They support the view that cognitive abilities of encoding, retrieval and probably spontaneous verbal mediation are compromised in elderly persons (Craik, 1977). Nevertheless, odors are known to be difficult to name and to identify whatever the age (Engen, 1982), and even a well-encoded highly familiar odor may fail to sufficiently activate its label in semantic memory (Lawless and Engen, 1977; Engen, 1987). Thus, differences in memory performances between both groups cannot be explained by a difference to identify odorants during encoding, since these differences are small between these two ranges of age (Doty et al., 1984). Finally, the subjects performed just a detection task during the encoding and were not instructed about the objective of the second session. Our previous data have revealed that the detection task does not activate the semantic neural networks (Royet et al., 1999, 2001). Thus, differences in semantic memory between both groups were difficult to conceive, particularly by taking into account the time allowed.

\section{CONCLUSION}

Although we did not explore familiarity and recollection via a remember-know procedure, but used a yes-no-recognition task and, our data are consistent with the hypothetical functional organization for memory processing (Eichenbaum et al., 2007) because the hippocampus and the parahippocampal gyrus were preferentially activated with true memory of odors (supposed to involve recollection), and the perirhinal cortex was more associated with false recognition (supposed to involve familiarity). However, we also observed that hippocampal activation was 
Table 6 | Cerebral areas activated in Hits, Misses, Correct rejections (CR) and false alarms (FA) and associated positively (+) or negatively (-) with bias measure.

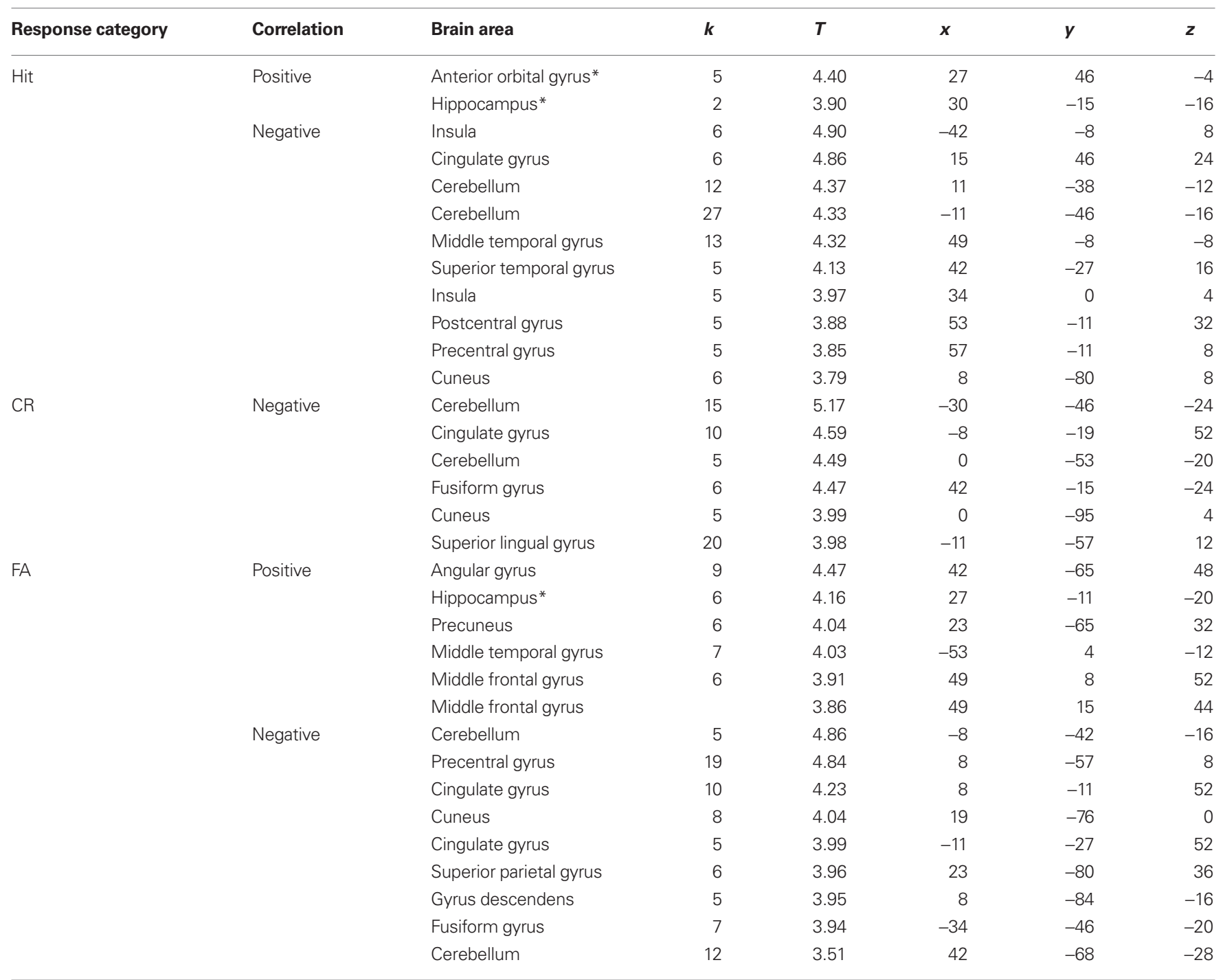

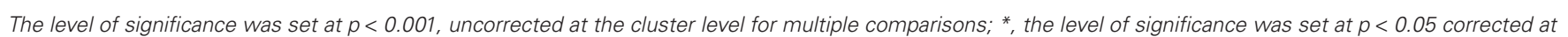
the voxel level for multiple comparisons, using small-volume correction (Worsley et al., 1996). Abbreviations: see Table 2.

negatively correlated with memory scores during Hit and CR responses, demonstrating less hippocampus involvement when recognition memory performance was high. We further demonstrated that processes differed between subject groups. Elderly individuals were more prone to false memories, and neuronal activation patterns were much less segregated than in young subjects. Activation in the hippocampus and the parahippocampal gyrus was positively correlated with response bias scores for Hit and FA, demonstrating that conservative subjects produced an additional search effort involving these two medial temporal lobe regions.

\section{REFERENCES}

Cabeza, R., Daselaar, S. M., Dolcos, F., Prince, S. E., Budde, M., and Nyberg, L. (2004). Task-independent and taskspecific age effects on brain activity during working memory, visual attention and episodic retrieval. Cereb. Cortex 14, 364-375.

Cabeza, R., Rao, S.M.,Wagner,A.D., Mayer, A. R., and Schacter, D. L. (2001). Can

\section{ACKNOWLEDGMENTS}

We thank all the members of CERMEP (especially D. Ibarrola, C. Vighi and F. Vey) for their invaluable assistance. We are also greatly indebted to S. Garcia for conceiving the software (with Python) to analyze behavioral and breathing data. This work was supported by the French National Institute for Agricultural Research (INRA) with the project PRA-05 (SIFOOD) to JeanPierre Royet, Léri Morin-Audebrand, Sylvie Issanchou, and Claire Sulmont-Rossé, the French National Research Agency with the project ANR-06-PNRA (AROMALIM) and NIH Grant R01AG04085 from the National Institute on Aging to Claire Murphy.

medial temporal lobe regions distinguish true from false? An event-related functional MRI study of veridical and illusory recognition memory. Proc. Natl. Acad. Sci. U.S.A. 98, 4805-4810.
Cerf-Ducastel, B., and Murphy, C. (2006). Neural substrates of cross-modal olfactory recognition memory: an fMRI study. Neuroimage 31, 386-396. 
Cerf-Ducastel, B., and Murphy, C. (2009). Age-related differences in the neural substrates of cross-modal olfactory recognition memory: an fMRI investigation. Brain Res. 1285, 88-98.

Chua, E. F., Schacter, D. L., RandGiovannetti, E., and Sperling, R. A. (2006). Understanding metamemory: neural correlates of the cognitive process and subjective level of confidence in recognition memory. Neuroimage 29, 1150-1160.

Ciaramelli, E., Grady, C. L., and Moscovitch, M. (2008). Top-down and bottom-up attention to memory: a hypothesis (AtoM) on the role of the posterior parietal cortex in memory retrieval. Neuropsychologia $46,1828-1851$.

Corwin, J. (1989). Olfactory identification in hemodialysis: acute and chronic effects on discrimination and response bias. Neuropsychologia 27, 513-522.

Craik, F. I. M. (1977). "Age differences in human memory," in Handbook of the Psychology of Aging, eds J.E. Birren and K.W.Schaie (New York: Van Nostrand Reinhold), 384-420.

Daselaar, S. M., Fleck, M. S., and Cabeza, R. (2006). Triple dissociation in the medial temporal lobes: recollection, familiarity, and novelty. $J$. Neurophysiol. 96, 1902-1911.

Daselaar, S. M., Rombouts, S. A., Veltman, D. J., Raaijmakers, J. G., Lazeron, R. H., and Jonker, C. (2001). Parahippocampal activation during successful recognition of words: a self-paced event-related fMRI study. Neuroimage 13, 1113-1120.

Davis, S. W., Dennis, N. A., Daselaar, S. M., Fleck, M. S., and Cabeza, R. (2008). Que PASA? The posterioranterior shift in aging. Cereb. Cortex 18, 1201-1209.

Dennis, N. A., Kim, H., and Cabeza, R. (2008). Age-related differences in brain activity during true and false memory retrieval. J. Cogn. Neurosci. 20, 1390-1402.

Doty, R. L., Shaman, P., Appelbaum, S. L., Giberson, R., Siksorski, L., and Rosenberg, L. (1984). Smell identification: changes with age. Science 226, 1441-1442.

Duarte, A., Ranganath, C., and Knight, R. T. (2005). Effects of unilateral prefrontal lesions on familiarity, recollection, and source memory. J. Neurosci. 25, 8333-8337.

Duvernoy, H. M. (1999). The Human Brain Surface, Three Dimentional Sectional Anatomy and MRI. New York: SpringerWien.

Eichenbaum, H., Yonelinas, A. P., and Ranganath, C. (2007). The medial temporal lobe and recognition memory. Annu. Rev. Neurosci. 30, 123-152.
Engen, T. (1982). The Perception of Odors. New York: Academic Press.

Engen, T. (1987). Remembreing odors and their names. Am. Sci. 75, 497-503.

Frankland, P. W., and Bontempi, B. (2005). The organization of recent and remote memories. Nat. Rev. Neurosci. 6, 119-130.

Friston, K. J., Ashburner, J., Frith, C., Poline, J. B., Healther, J. D., and Frackowiak, R. S. (1995a). Spatial registration and normalization of images. Hum. Brain Mapp. 2, 165-189.

Friston, K. J., Holmes, A. P., Worsley, K. J., Poline, J. B., Frith, C., and Frackowiak, R.S. (1995b).Statistical parametric maps in functional imaging: a general linear approach. Hum. Brain Mapp.2, 189-210.

Friston, K. J., Josephs, O., Rees, G., and Turner, R. (1998). Nonlinear eventrelated responses in fMRI. Magn. Reson. Med. 39, 41-52.

Friston, K. J., Poline, J. B., Holmes, A. P., Frith, C. D., and Frackowiak, R. S. (1996). A multivariate analysis of PET activation studies. Hum. Brain Mapp. 4, 140-151.

Garoff-Eaton, R. J., Slotnick, S. D., and Schacter, D. L. (2006). Not all false memories are created equal: the neural basis of false recognition. Cereb. Cortex 16, 1645-1652.

Goldmann, R. E., Sullivan, A. L., Droller, D. B., Rugg, M. D., Curran, T., Holcomb, P. J., Schacter, D. L., Daffner, K. R., and Budson, A. E. (2003). Late frontal brain potentials distinguish true and false recognition. Neuroreport 14, 1717-1720.

Gottfried, J. A. (2010). Central mechanisms of odour object perception. Nat. Rev. Neurosci. 11, 628-641.

Gottfried, J.A., Deichmann, R., Winston, J. S., and Dolan, R. J. (2002). Functional heterogeneity in human olfactory cortex: an event-related functional magnetic resonance imaging study. J. Neurosci. 22, 10819-10828.

Gottfried, J. A., Smith, A. P., Rugg, M. D., and Dolan, R. J. (2004). Remembrance of odors past: human olfactory cortex in cross-modal recognition memory. Neuron 42, 687-695.

Gottfried, J.A., and Zald, D. H. (2005). On the scent of human olfactory orbitofrontal cortex: meta-analysis and comparison to non-human primates. Brain Res. Brain Res. Rev. 50, 287-304.

Heun, R., Jessen, F., Klose, U., Erb, M., Granath, D. O., and Grodd, W. (2004). Response-related fMRI of veridical and false recognition of words. Eur. Psychiatry 19, 42-52.

Hopfinger, J. B., Buchel, C., Holmes, A. P., and Friston, K. J. (2000). A study of analysis parameters that influence the sensitivity of event-related fMRI analyses. Neuroimage 11, 326-333.
Jacoby, L. L. (1991). A process dissociation framework: separating automatic from intentional uses of memory. $J$. Mem. Lang. 30, 513-626.

Kim, H., and Cabeza, R. (2007). Trusting our memories: dissociating the neural correlates of confidence in veridical versus illusory memories. J. Neurosci. 27, 12190-12197.

Lawless, H., and Engen, T. (1977). Associations to odors: interference, mnemonics, and verbal labeling. $J$. Exp. Psychol. Hum. Learn. 3, 52-59.

Leube, D. T., Erb, M., Grodd, W., Bartels, M., and Kircher, T. T. (2003). Successful episodic memory retrieval of newly learned faces activates a left fronto-parietal network. Brain Res. Cogn. Brain Res. 18, 97-101.

Lockhart, R. S., and Murdock, B. B. (1970) Memory and the theory of signal detection. Psychol. Bull. 74, 100-109.

Mai, J. K., Paxinos, G., and Voss, T. (2008). Atlas of the Human Brain. San Diego, CA: Academic Press.

Montaldi, D., Spencer, T. J., Roberts, N., and Mayes, A. R. (2006). The neural system that mediates familiarity memory. Hippocampus 16, 504-520.

Moritz, S., Glascher, J., Sommer, T., Buchel, C., and Braus, D. F. (2006). Neural correlates of memory confidence. Neuroimage 33, 1188-1193.

Murphy, C., Nordin, S., and Acosta, L. (1997). Odor learning, recall, and recognition memory in young and elderly adults. Neuropsychology 11 , 126-137.

Plailly, J., Delon-Martin, C., and Royet, J. P. (2011). Experience induces functional reorganization in brain regions involved in odor imagery in perfumers. Hum. Brain Mapp. doi: 10.1002/ hbm.21207. [Epub ahead of print].

Plailly, J., Howard, J. D., Gitelman, D. R., and Gottfried, J. A. (2008). Attention to odor modulates thalamocortical connectivity in the human brain. $J$. Neurosci. 28, 5257-5267.

Plailly, J., Tillmann, B., and Royet, J. P. (2007). The feeling of familiarity of music and odors: the same neural signature? Cereb. Cortex 17, 2650-2658.

Quamme, J. R., Weiss, D. J., and Norman, K. A. (2010). Listening for recollection: a multi-voxel pattern analysis of recognition memory retrieval strategies. Front. Hum. Neurosci. 4:61. doi: 10.3389/fnhum.2010.00061

Roediger, H. L. I., and McDermott, K. B. (1995). Creating false memories: remebering words not presented in lists. J. Exp. Psychol. Learn. Mem. Cogn. 21, 803-814.

Royet, J. P., Hudry, J., Zald, D. H., Godinot, D., Gregoire, M. C., Lavenne, F., Costes, N., and Holley, A. (2001). Functional neuroanatomy of different olfactory judgments. Neuroimage 13, 506-519.
Royet, J. P., Koenig, O., Gregoire, M. C., Cinotti, L., Lavenne, F., Le Bars, D., Costes, N., Vigouroux, M., Farget, V., Sicard, G., Holley, A., Mauguiere, F., Comar, D., and Froment, J. C. (1999). Functional anatomy of perceptual and semantic processing for odors. J. Cogn. Neurosci. 11, 94-109.

Royet, J. P., Plailly, J., Delon-Martin, C., Kareken, D. A., and Segebarth, C. (2003). fMRI of emotional responses to odors: influence of hedonic valence and judgment, handedness, and gender. Neuroimage 20, 713-728.

Savic, I., and Berglund, H. (2004). Passive perception of odors and semantic circuits. Hum. Brain Mapp. 21, 271-278.

Savic, I., Gulyas, B., Larsson, M., and Roland,P.(2000). Olfactory functions are mediated by parallel and hierarchical processing. Neuron 26, 735-745.

Slotnick, S. D., and Schacter, D. L. (2004). A sensory signature that distinguishes true from false memories. Nat. Neurosci. 7, 664-672.

Snodgrass, J. G., and Corwin, J. (1988). Pragmatics of measuring recognition memory: applications to dementia and amnesia. J. Exp. Psychol. Gen. 117, 34-50.

Stark, C. E., and Squire, L. R. (2001). When zero is not zero: the problem of ambiguous baseline conditions in fMRI. Proc. Natl. Acad. Sci. U.S.A. 98, 12760-12766.

Vigouroux, M., Bertrand, B., Farget, V., Plailly, J., and Royet, J. P. (2005). A stimulation method using odors suitable for PET and fMRI studies with recording of physiological and behavioral signals. J. Neurosci. Methods 142, 35-44.

Vilberg, K. L., and Rugg, M. D. (2008). Memory retrieval and the parietal cortex: a review of evidence from a dualprocess perspective. Neuropsychologia 46, 1787-1799.

Wais, P. E., Squire, L. R., and Wixted, J. T. (2010). In search of recollection and familiarity signals in the hippocampus. J. Cogn. Neurosci. 22, 109-123.

Wixted, J. T., and Stretch, V. (2004). In defense of the signal detection interpretation of remember/know judgments. Psychon. Bull. Rev. 11,616-641.

Worsley, K. J., Marrett, S., Neelin, P., Vandal, A. C., Friston, K. J., and Evans, A. C. (1996). A unified statistical approach for determining significant signals in images of cerebral activation. Hum. Brain Mapp. 4, 58-73.

Yonelinas, A. P. (1994). Receiver-operating characteristics in recognition memory: evidence for a dual-process model. J. Exp. Psychol. Learn Mem. Cogn. 20, 1341-1354.

Yonelinas, A. P., Dobbins, I., Szymanski, M. D., Dhaliwal, H. S., and King, L. (1996). Signal-detection, threshold, 
and dual-process models of recognition memory: ROCs and conscious recollection. Conscious. Cogn. 5, 418-441.

Yonelinas, A. P., Otten, L. J., Shaw, K. N., and Rugg, M. D. (2005). Separating the brain regions involved in recollection and familiarity in recognition memory. J. Neurosci. 25, 3002-3008.
Conflict of Interest Statement: The authors declare that the research was conducted in the absence of any commercial or financial relationships that could be construed as a potential conflict of interest.

Received: 01 April 2011; accepted: 08 July 2011; published online: 21 July 2011.
Citation: Royet J-P, Morin-Audebrand L, Cerf-Ducastel B, Haase L, Issanchou S, Murphy C, Fonlupt P, Sulmont-Rossé C and Plailly J (2011) True and false recognition memories of odors induce distinct neural signatures. Front. Hum. Neurosci. 5:65. doi: 10.3389/fnhum.2011.00065 Copyright (C) 2011 Royet, MorinAudebrand, Cerf-Ducastel, Haase,
Issanchou, Murphy, Fonlupt, SulmontRossé and Plailly. This is an open-access article subject to a non-exclusive license between the authors and Frontiers Media $S A$, which permits use, distribution and reproduction in other forums, provided the original authors and source are credited and other Frontiers conditions are complied with. 